\title{
ALS-associated mutation FUS-R521C causes DNA damage and RNA splicing defects
}

\author{
Haiyan Qiu,, ${ }^{1,2}$ Sebum Lee, ${ }^{1,2}$ Yulei Shang, ${ }^{1,2}$ Wen-Yuan Wang, ${ }^{3}$ Kin Fai Au, ${ }^{4}$ Sherry Kamiya, ${ }^{1,2}$ \\ Sami J. Barmada, ${ }^{5}$ Steven Finkbeiner, ${ }^{5}$ Hansen Lui,, ${ }^{1,2}$ Caitlin E. Carlton, ${ }^{1,2}$ Amy A. Tang, ${ }^{1,2}$ \\ Michael C. Oldham, ${ }^{6}$ Hejia Wang, ${ }^{7}$ James Shorter, ${ }^{7}$ Anthony J. Filiano, ${ }^{8}$ Erik D. Roberson, ${ }^{8}$ \\ Warren G. Tourtellotte, ${ }^{9}$ Bin Chen, ${ }^{10}$ Li-Huei Tsai, ${ }^{3}$ and Eric J. Huang ${ }^{1,2}$ \\ 1Department of Pathology, UCSF, San Francisco, California, USA. 2Pathology Service, VA Medical Center, San Francisco, California, USA. \\ ${ }_{3}^{3}$ Picower Institute for Learning and Memory and Howard Hughes Medical Institute, Massachusetts Institute of Technology, Cambridge, Massachusetts, USA. \\ ${ }^{4}$ Department of Statistics, Stanford University, Stanford, California, USA. ${ }^{5}$ Gladstone Institute of Neurological Disease, San Francisco, California, USA. \\ ${ }^{6}$ The Eli and Edythe Broad Center of Regeneration Medicine and Stem Cell Research, UCSF, San Francisco, California, USA. \\ ${ }^{7}$ Department of Biochemistry and Biophysics, University of Pennsylvania, Philadelphia, Pennsylvania, USA. ${ }^{8}$ Department of Neurology, \\ University of Alabama at Birmingham, Birmingham, Alabama, USA. ${ }^{D}$ Department of Pathology, Northwestern University, Chicago, Illinois, USA \\ ${ }^{10}$ Department of Molecular Cellular and Developmental Biology, University of California Santa Cruz, Santa Cruz, California, USA
}

\begin{abstract}
Autosomal dominant mutations of the RNA/DNA binding protein FUS are linked to familial amyotrophic lateral sclerosis (FALS); however, it is not clear how FUS mutations cause neurodegeneration. Using transgenic mice expressing a common FALS-associated FUS mutation (FUS-R521C mice), we found that mutant FUS proteins formed a stable complex with WT FUS proteins and interfered with the normal interactions between FUS and histone deacetylase 1 (HDAC1). Consequently, FUS-R521C mice exhibited evidence of DNA damage as well as profound dendritic and synaptic phenotypes in brain and spinal cord. To provide insights into these defects, we screened neural genes for nucleotide oxidation and identified brain-derived neurotrophic factor $(B d n f)$ as a target of FUS-R521C-associated DNA damage and RNA splicing defects in mice. Compared with WT FUS, mutant FUS-R521C proteins formed a more stable complex with Bdnf RNA in electrophoretic mobility shift assays. Stabilization of the FUS/Bdnf RNA complex contributed to $B d n f$ splicing defects and impaired BDNF signaling through receptor TrkB. Exogenous BDNF only partially restored dendrite phenotype in FUS-R521C neurons, suggesting that BDNF-independent mechanisms may contribute to the defects in these neurons. Indeed, RNA-seq analyses of FUS-R521C spinal cords revealed additional transcription and splicing defects in genes that regulate dendritic growth and synaptic functions. Together, our results provide insight into how gain-of-function FUS mutations affect critical neuronal functions.
\end{abstract}

\section{Introduction}

Autosomal dominant mutations in RNA/DNA binding protein fused in sarcoma/translocated in liposarcoma (FUS/TLS) have been causally linked to familial ALS (FALS). The main pathological features in FALS with FUS mutations are FUS-positive protein aggregates in neuronal cytoplasm and dendrites $(1,2)$. While the majority of these aggregates are identified in the spinal motor neurons, FUS-positive aggregates have also been found in neurons in cerebral cortex and brainstem nuclei $(3,4)$, raising the possibility that FUS mutations may have a broader impact on the functions of other neuronal subtypes. Consistent with these observations, a subset of FALS-FUS patients also exhibits cognitive impairments during the developmental or degenerative processes.

Although the exact mechanism of FUS mutations remains unclear, several previous studies have provided compelling evidence that FUS can regulate DNA damage response, transcription, and RNA processing. For instance, fibroblasts and lymphocytes from fus-null mice show increased sensitivity to ionizing irradiation and genomic instability, respectively $(5,6)$. Consistent with these results, our recent study shows that WT FUS proteins are rapidly recruited to DNA damage foci in neurons, where it interacts with histone deacetylase 1 (HDAC1), a critical component in

Authorship note: Haiyan Qiu and Sebum Lee contributed equally to this work. Conflict of interest: The authors have declared that no conflict of interest exists. Citation for this article: J Clin Invest. 2014;124(3):981-999. doi:10.1172/JCI72723.
DNA damage response and repair machinery $(7,8)$. Interestingly, FUS proteins that harbor human FALS mutations show diminished interaction with HDAC1 and are defective in DNA damage response and repair (7). Furthermore, FALS patients with FUSR521C or FUS-P525L mutation exhibit evidence of DNA damage in cortical neurons and spinal motor neurons.

These results provide critical mechanistic insights to neurodegeneration caused by FUS mutations because DNA damage is known to affect transcription by regulating the activity of RNA polymerase II and the ensuing RNA processing, including alternative splicing (9-11). Indeed, transcription of RNA in the eukaryotic cells is a dynamic and tightly regulated process that involves multiple intricately connected steps, including splicing of pre-mRNA and transport of mature mRNA to its final destinations $(12,13)$. In the nervous system, these regulatory mechanisms are known to generate a vast diversity of RNA transcripts that control cell fate determination, axon guidance, dendritic growth, and synaptic functions $(14,15)$. Perturbations to these critical mechanisms have been implicated in neuromuscular diseases, neurodevelopmental disorders, and neurodegenerative diseases (16).

The implications of FUS in RNA splicing are further underscored by the identification of FUS as one of the approximately 50 non-snRNP proteins in the pre-spliceosome (17). These results raise the possibility that FUS may regulate the recognition of the $5^{\prime}$ splice junction, the formation and stability of complex A, and the efficiency of splicing. Recent studies using cross-linking and 
A

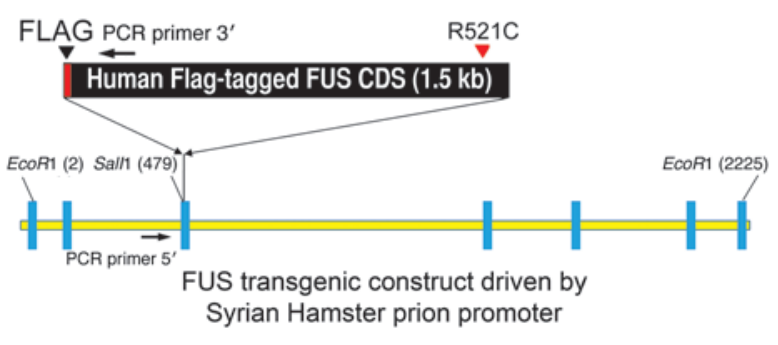

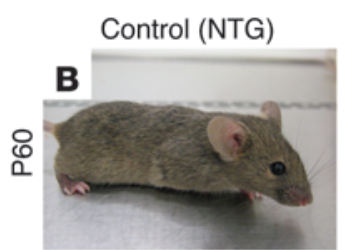

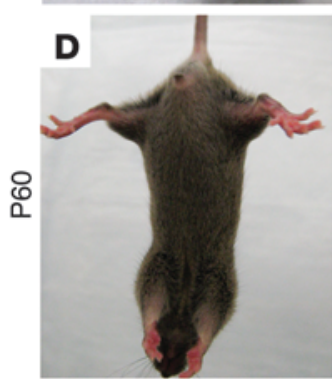

FUS-R521C
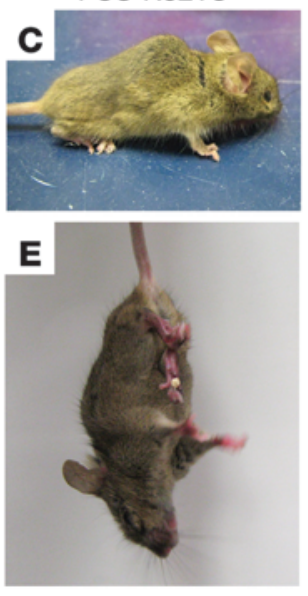

F

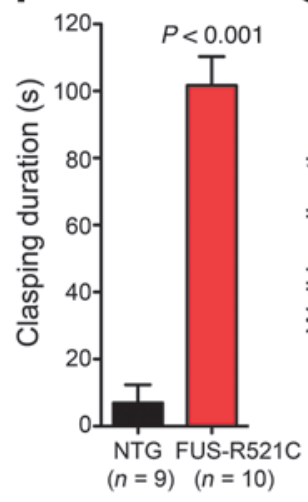

G

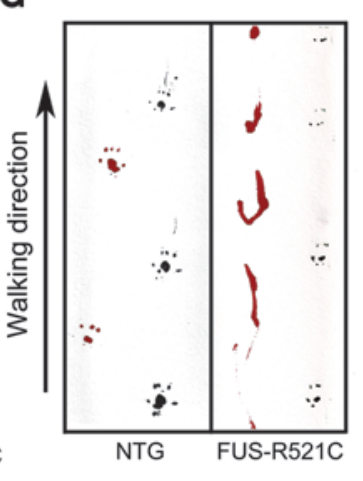

H

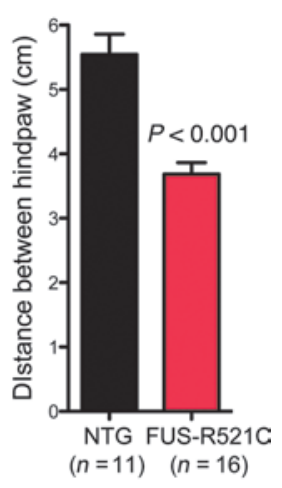

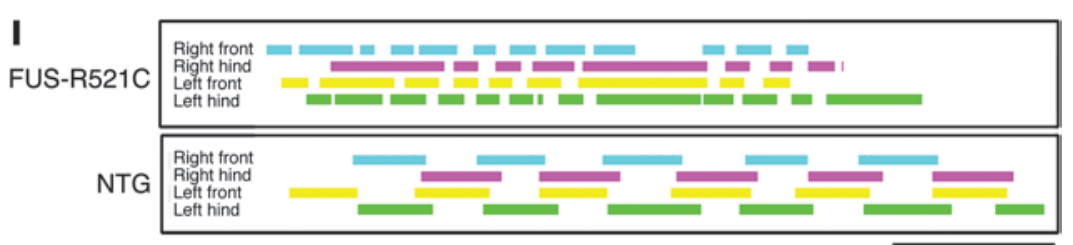

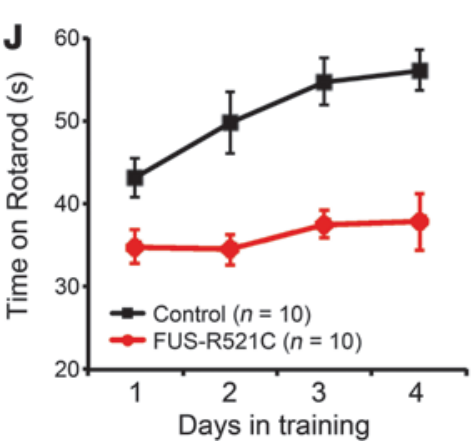

$10 \mathrm{~cm}$

\section{Figure 1}

Early onset motor behavioral deficits and postnatal lethality in FUS-R521C transgenic mice. (A) A schematic diagram showing our strategy for generating transgenic mice that express human FUS-R521C mutant proteins using the Syrian hamster prion promoter. (B and C) The majority of N1F1 FUS-R521C mice show growth retardation, spastic paraplegia, and severe muscle wasting. (D and E) In addition, the FUS-R521C mice also exhibit prolonged hind limb clasping on tail-suspension test. (F) In a 2-minute period, FUS-R521C mice spend approximately 100 seconds with hind limbs clasped together, whereas nontransgenic control mice exhibit similar clasping phenotype for less than 10 seconds. 2-tailed Student's $t$ test, $P<0.001$. ( $\mathbf{G}$ and $\mathbf{H}$ ) Due to the spastic paraplegia, FUS-R521C mice show reduced distance between hind paws during tandem walk tests. 2-tailed Student's $t$ test, $P<0.001$. (I) The gait and motor coordination defects in FUS-R521C mice are further verified using computerized catwalk measurements. (J) In addition to the gait abnormalities, FUS-R521C N1F1 mice show poor performance and motor learning on rotarod tests.

immunoprecipitation (CLIP) identify many potential RNA targets for FUS in HEK293 cells and brain tissue, most of which are intronic sequences $(18,19)$. Remarkably, FALS-related mutation FUS-R521G exhibits a drastic shift in binding preference from the intronic sequences to sequences in the $3^{\prime}$ UTR (18), suggesting that mutations in FUS can potentially alter its interaction with RNA targets. Together, these results suggest that, in addition to DNA damage response and repair, FALS-associated FUS mutations may have broader effects on transcription and RNA processing.

Here, we report that transgenic mice expressing FUS-R521C, a common mutation in FALS-FUS patients, showed severe dendritic and synaptic defects in spinal motor neurons and cortical neurons. In agreement with results from human patients (7), FUS-R521C transgenic mice exhibited evidence of DNA damage in cortical neurons and spinal motor neurons. By screening a number of neural genes implicated in DNA damage during neuro- degeneration $(20,21)$, we found that the FUS-R521C mice exhibited DNA damage repair defects in the $5^{\prime}$ noncoding exons of the brain-derived neurotrophic factor $(B d n f)$ gene, which contained transcriptional start sites and required splicing of long intronic sequences to generate mature $B d n f$ mRNA. Consistent with this idea, we found retentions of $5^{\prime}$ splice junctions in the $B d n f$ mRNA in FUS-R521C mice and defects in transporting $B d n f$ mRNA to distal dendrites due to the abnormal increase in the binding of FUSR521C to splice junction and the $3^{\prime}$ UTR sequences of Bdnf premRNA. Finally, genome-wide survey using RNA-seq transcriptome analyses revealed defects in the transcription and RNA processing of additional genes that are critical for dendritic growth and synaptic functions. Together, our results unraveled previously unrecognized gain-of-function properties of FUS-R521C mutation in DNA damage repair and the transcription of genes required for dendritic growth and synaptic functions. 
A

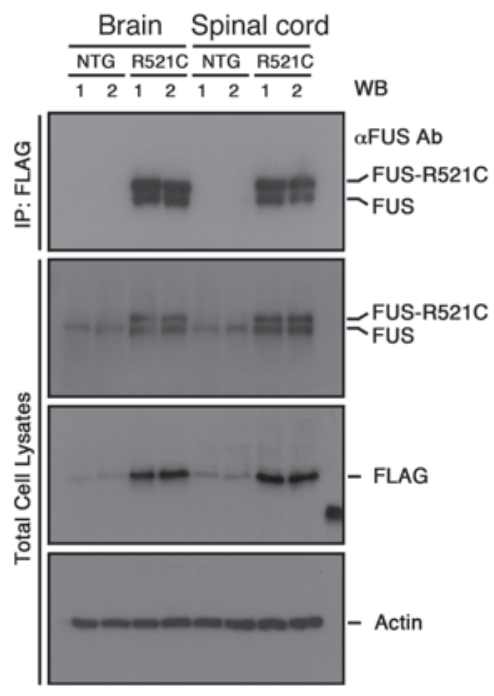

B

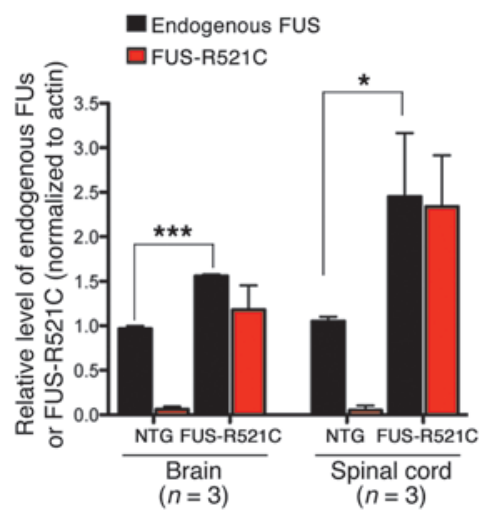

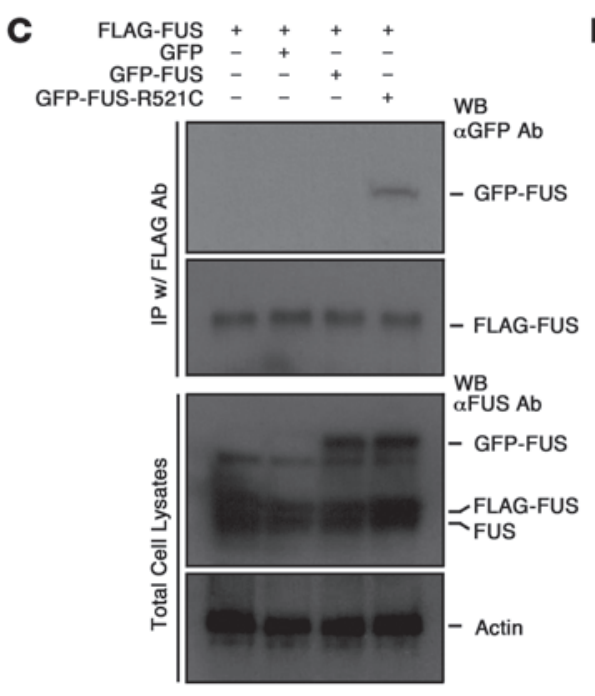

$\begin{array}{rrrrrr}\text { D FLAG-FUS-R521C } & + & + & + & & \\ \text { GFP } & - & + & - & - \\ \text { GFP-FUS } & - & - \\ \text { GFP-FUS-R521C } & - & - & - & + & \text { WB }\end{array}$
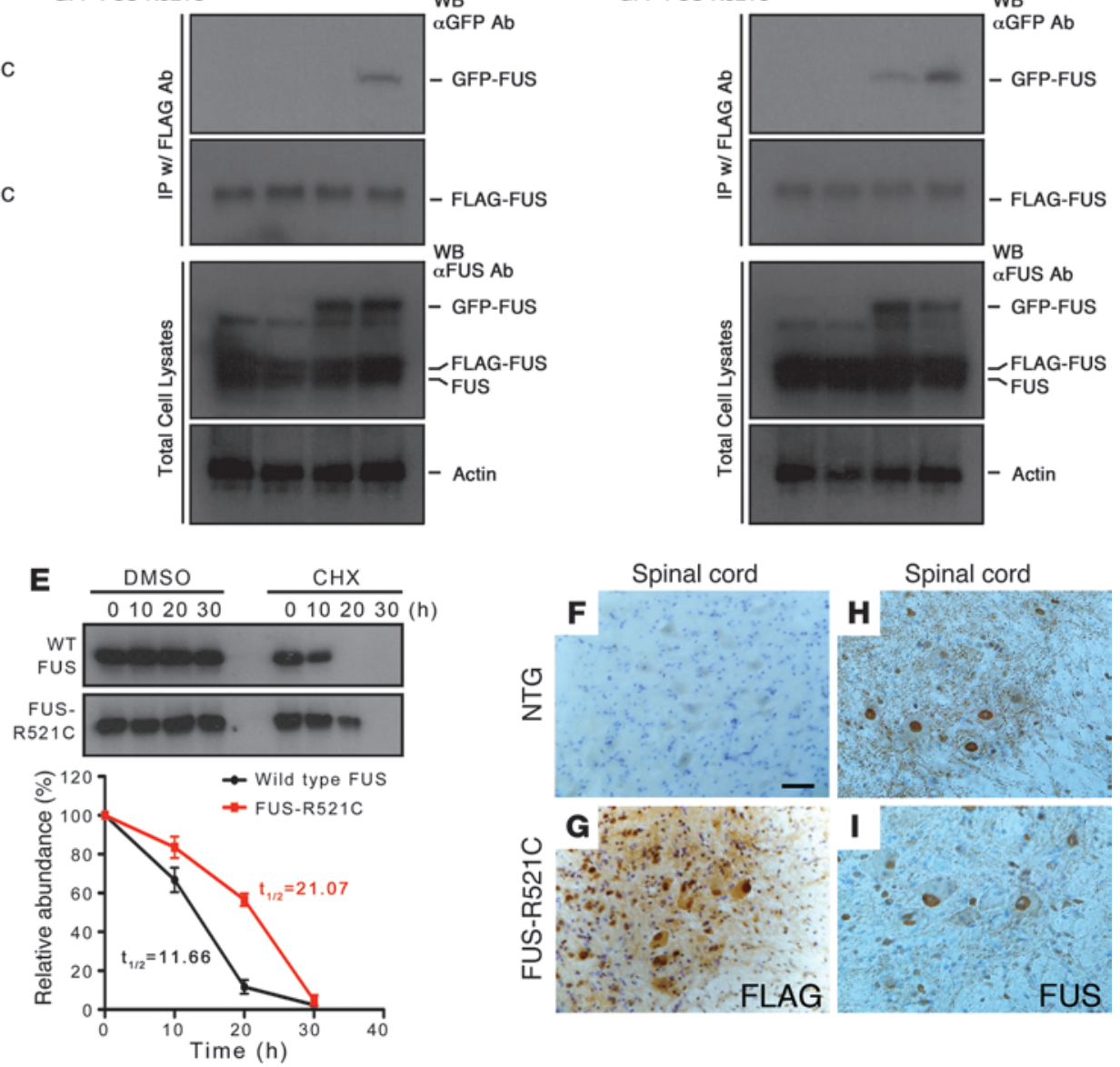
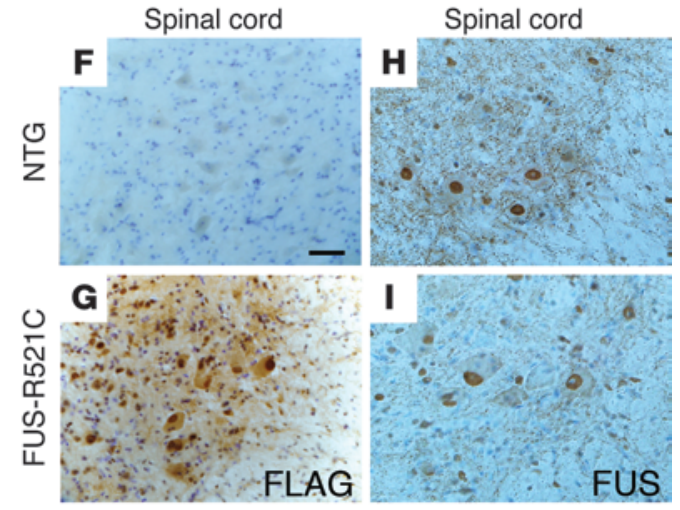

\section{Figure 2}

FUS-R521C interacts with the WT FUS and perturbs its distribution. (A and B) Western blots showing the level of FUS-R521C proteins is similar to that of the endogenous FUS proteins in 2 independent transgenic mice (P37). However, immunoprecipitation shows that FUS-R521C can be detected in a complex with the endogenous WT FUS. The relative levels of endogenous FUS and FUS-R521C in control (NTG) and FUS-R521C brain and spinal cord are quantified in B. Student's $t$ test, $n=3$ for each group. ${ }^{*} P<0.017$; ${ }^{* \star *} P<0.008$. (C and D) Coimmunoprecipitation assays using lysates from transfected HEK293T cells demonstrate that FUS-R521C has a higher propensity to form protein complexes with FUS-R521C proteins than with WT FUS proteins. (E) Pulse-chase experiments in HEK293T cells showing FUS-R521C proteins are twice as stable as WT FUS proteins. (F and G) IHC using FLAG antibody shows FUS-R521C proteins are predominantly found in the nuclei of spinal motor neurons, but can also be detected in the cytoplasm. (H and I) FUS proteins are found predominantly in the nuclei of spinal motor neurons and in punctate synaptic structures in the spinal cord. The punctate FUS staining in synapses is diminished in FUS-R521C mice (P37). Scale bar: $25 \mu \mathrm{m}$.

\section{Results}

FUS-R521C forms stable complex with, and perturbs the localization of, WT FUS proteins. To investigate the mechanisms of FUS mutations, we generated transgenic mice that expressed FLAG-tagged FUS-R521C mutant proteins using the Syrian hamster prion promoter (Figure 1A and ref. 22). The N1F1 transgenic mice exhibited neurological phenotypes, characterized by motor behavioral abnormalities, including severe spastic paraplegia, abnormal gaits, reduced muscle mass, and motor coordination problems (Figure 1, B-F). Most N1F1 transgenic mice died within 4 to 6 weeks after symptom onset (Supplemental Figure 1; supplemental material available online with this article; doi:10.1172/JCI72723DS1). For those that did survive, they exhibited motor symptoms, ranging from gait problems revealed by CatWalk apparatus to poor performances on rotarod tests (Figure 1, G-J). To propagate FUS-R521C transgenic line, 3- to 6-month-old surviving N1 mice were mated with $\mathrm{C} 57 \mathrm{BL} / 6$ mice to generate N2 progenies that developed similar disease onset and survival curves (Supplemental Figure 1).

Western blots analyses showed that the level of FUS-R521C proteins in the brain and spinal cord of N1F1 FUS-R521C mice (1 month old) was similar to that of the endogenous FUS proteins (Figure 2A). Interestingly, however, the levels of endogenous FUS proteins in FUS-R521C transgenic mice seemed increased compared with controls, raising the possibility that FUS-R521C mutant proteins may stabilize the WT FUS proteins via protein complex formation (Figure 2B and ref. 23). Consistent with this idea, immunoprecipitation using FLAG antibody detected the endogenous FUS proteins in complex with FUS-R521C mutant proteins (Figure $2 \mathrm{~A}$ ). To further characterize the potential interactions between WT FUS and mutant FUS-R521C proteins, we 
coexpressed FLAG-tagged WT FUS and GFP-tagged WT FUS, or FLAG-tagged WT FUS and GFP-tagged FUS-R521C in HEK293T cells. Using coimmunoprecipitation with anti-FLAG antibodies, we showed that GFP-tagged FUS-R521C proteins, but not GFP-tagged WT FUS proteins, could be detected in complexes with FLAG-tagged WT FUS (Figure 2C). Interestingly, coimmunoprecipitation using proteins from HEK293T cells expressing FLAG-tagged FUS-R521C or GFP-tagged WT FUS or FUS-R521C proteins showed that much more abundant FLAG-tagged FUSR521C proteins were in complex with GFP-tagged FUS-R521C (Figure 2D). These results demonstrated that FUS-R521C proteins had a higher propensity to form complexes with FUS-R521C than with WT FUS proteins. To directly determine whether mutant FUS-R521C proteins were indeed more stable, we treated HEK293T cells expressing WT FUS or FUS-R521C with cycloheximide (CHX) to block protein synthesis and determined the relative abundance of WT FUS and FUS-R521C proteins. Our results showed that the half-life $\left(t_{1 / 2}\right)$ for WT FUS in HEK293T cells was 11.66 hours, whereas $t_{1 / 2}$ for FUS-R521C was 21.07 hours, almost twice as stable as shown in WT FUS (Figure 2E). Together, these results supported the idea that FUS-R521C proteins were inherently more stable and had a high propensity to form protein complexes with WT FUS proteins.

To further determine how mutant FUS-R521C proteins might affect the endogenous FUS proteins, we performed immunohistochemistry (IHC) in nontransgenic littermate (hereafter referred to as NTG or control) and FUS-R521C transgenic brain and spinal cord tissues using FLAG and FUS antibodies. Whereas the FLAG IHC in control spinal cord showed no signals, the FLAG-tagged FUS-R521C proteins were detected predominantly in the nuclei of spinal motor neurons, with a relatively low abundance in the neuronal cytoplasm (Figure 2, F and G). Interestingly, in control spinal cord, the endogenous FUS proteins were detected in neuronal nuclei and in punctate structures that resembled synapse (Figure $2 \mathrm{H}$ and ref. 24 ), which were drastically reduced in the FUS-R521C tissues (Figure 2I), suggesting that FUS-R521C proteins may have affected the distribution of endogenous FUS at the nerve terminals. Similar reduction of $\mathrm{FUS}^{+}$punctate was also observed in the sensorimotor cortex (data not shown). In addition to motor neurons, we found that the endogenous FUS in control spinal cord could be detected in astrocytes and oligodendroglia, but not in microglia. Similar to the endogenous FUS, FLAG-tagged FUS-R521C could also be detected in astrocytes and oligodendroglia, but not in microglia of FUS-R521C mice (Supplemental Figure 2).

FUS-R521C mice show evidence of DNA damage in cortical and spinal motor neurons. The presence of a more stable protein complex between FUS-R521C and endogenous WT FUS proteins suggested that mutations in FUS might alter its ability to interact with other cofactors. Indeed, our recent results indicate that FUS-R521C mutant proteins show a diminished interaction with HDAC1, an important chromatin remodeling agent critical for DNA damage response and repair (7). To determine whether the presence of FUSR521C could also interfere with FUS interaction with HDAC1 in vivo, we characterized the interaction of FUS and HDAC1 using protein lysates from cortex and spinal cord of FUS-R521C mice. Coimmunoprecipitation using anti-FUS or anti-HDAC1 antibodies and Western blotting using the reciprocal antibodies showed that the interaction between HDAC1 and the endogenous FUS in the FUS-R521C mice was diminished compared with the robust interaction detected in the control littermates (Figure 3A). These results suggested that FUS-R521C mutant proteins not only failed to interact with HDAC1, they could also interfere with the interaction between WT FUS and HDAC1.

To determine whether FUS-R521C mice also exhibited DNA damage phenotypes similar to those observed in human patients, we evaluated levels of DNA damage in the nervous system of FUS-R521C mice by immunoblotting for $\gamma \mathrm{H} 2 \mathrm{AX}$ in the cortex and spinal cord. Whereas control littermates showed no detectable $\gamma \mathrm{H} 2 \mathrm{AX}$, FUS-R521C mice exhibited notably increased $\gamma \mathrm{H} 2 \mathrm{AX}$ levels in both the cortex and spinal cord (Figure 3B), indicating increased DNA damage. These results were confirmed with IHC in sections from the cortex and spinal cord of FUS-R521C and control mice (Figure 3, C and D). Using confocal microscopy, we showed that the majority of $\gamma \mathrm{H} 2 \mathrm{AX}$ signals were present in choline acetyltransferase-positive $\left(\mathrm{ChAT}^{+}\right)$spinal motor neurons (Figure $3 \mathrm{C})$. However, there were few fragmented $\gamma \mathrm{H} 2 \mathrm{AX}$ signals in the anterior horn of FUS-R521C spinal cord that could be present in either dead neurons or in glial nuclei (Figure 3C). In addition to $\gamma \mathrm{H} 2 \mathrm{AX}$, we also determined the expression of ATF3, which could be activated upon DNA damage (25). In line with the elevated $\gamma \mathrm{H} 2 \mathrm{AX}$ signal, we also found increased ATF3 levels in mutant tissues by Western blot (Figure 3B). Thus, these supported the presence of DNA damage in cortical and spinal motor neurons of FUSR521C transgenic mice. To directly assess the presence of DNA damage in FUS-R521C mice, we performed comet assays using neurons isolated from FUS-R521C mice and littermate controls. We found that more than $50 \%$ of the neurons from FUS-R521C mice demonstrated comet tails, compared with approximately $20 \%$ of neurons from control mice (Figure 3E). Together, these findings were similar to the results in patients with FUS-R521C mutation and supported that FUS-R521C transgenic mice could be a valid model that further elucidates the mechanisms of neurodegeneration in FALS caused by FUS mutations.

Dendritic and synaptic defects in spinal motor neurons of FUS-R521C mice. To determine how FUS-R521C proteins might affect the survival of spinal motor neurons, we used ChAT immunostaining to quantify the number of spinal motor neurons in FUS-R521C mice from perinatal stages to disease end-stage (Figure 4, A and B). At P3, motor neurons in FUS-R521C mice showed no detectable reduction. However, at P16, FUS-R521C mice showed a modest $20 \%$ reduction in $\mathrm{ChAT}^{+}$motor neurons, and by end-stage, typically between the ages of 1 and 3 months, FUS-R521C mice showed approximately 55\% loss of motor neurons (Supplemental Figure 3, $A$ and $B$ ), and the majority of the neuromuscular junctions (NMJ) in the diaphragm of the FUS-R521C mice showed partial or complete loss of innervation (Supplemental Figure 3, C-E). These motor neuron loss results were distinctly different from those in SOD1 $1^{\mathrm{G} 93 A}$ mice, in which less than $10 \%$ of spinal motor neurons survive by disease end-stage $(26,27)$. Unlike the SOD $1^{\mathrm{G} 93 A}$ mice, we could not detect cleaved caspase 3 - or TUNEL-positive neurons in the FUS-R521C spinal cord (data not shown). Instead, we found a prominent increase of microgliosis and a modest astrogliosis in the anterior horn of FUS-R521C mice (Supplemental Figure 3, F-I), which may contribute to motor neuron loss through increased phagocytic activity.

In light of the role of FUS in regulating dendritic spine morphology (24), we asked whether mutant FUS-R521C proteins might interfere with the maintenance of dendritic arborization and synaptic structures. To test this, we performed Golgi staining 

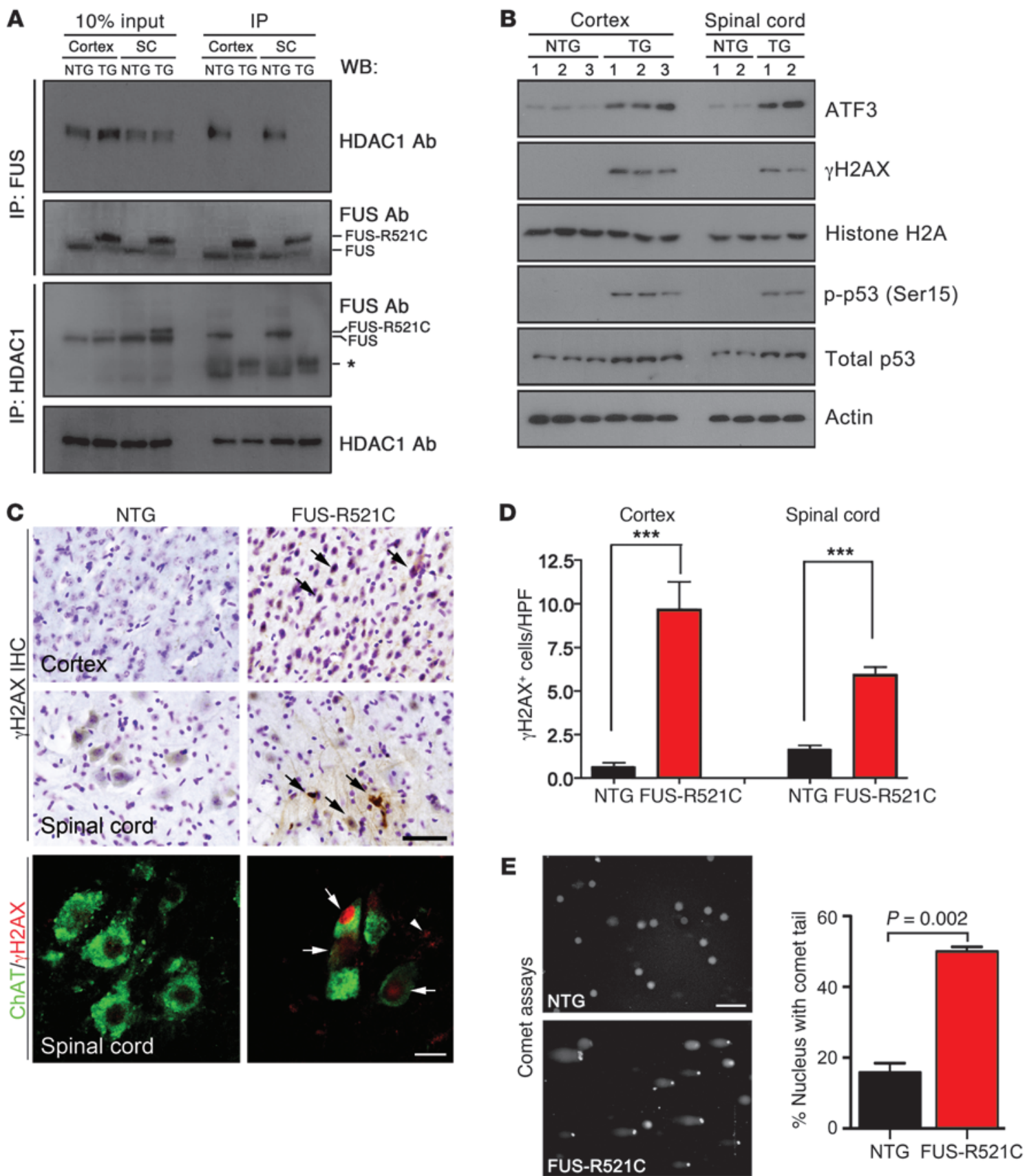

\section{Figure 3}

FUS-R521C transgenic mice exhibit an impairment of FUS/HDAC1 interaction and an increase in DNA damage. (A) Immunoprecipitation and Western blotting with the indicated antibodies of protein lysates from the control cortex and spinal cord show WT FUS in complex with HDAC1. However, protein lysates from FUS-R521C mutants show no evidence of protein complexes between FUS and HDAC1 or between FUSR521C and HDAC1. Asterisk indicates nonspecific proteins that crossreact with FUS antibody. (B) Western blot analysis shows increased $\gamma \mathrm{H} 2 \mathrm{AX}$, ATF3, and phosphorylated p53 levels in protein lysates from cortex and spinal cord of FUS-R521C transgenic mice. NTG, control nontransgenic mice; TG: transgenic mice. (C) DAB staining and immunofluorescent images of $\gamma \mathrm{H} 2 \mathrm{AX}$ (arrows) in cortex and spinal cord in control and FUS-R521C mice. The confocal images (bottom) show that most $\gamma \mathrm{H} 2 \mathrm{AX}+$ staining is detected in spinal motor neurons (arrows), but smaller foci of $\gamma \mathrm{H} 2 \mathrm{AX}$ can also be detected in dying neurons or glial nuclei (arrowhead). Scale bars: $50 \mu \mathrm{m}(\gamma \mathrm{H} 2 \mathrm{AX}$ IHC panel); $5 \mu \mathrm{m}$ (ChAT/ $\gamma \mathrm{H} 2 \mathrm{AX}$ IF). (D) The number of $\gamma \mathrm{H} 2 \mathrm{AX}$-positive cells per high-power field in FUS-R521C transgenic mice is increased compared with NTG mice. $n=3$ mice, 7-9 sections per mouse (mean \pm SEM, ${ }^{* * *} P<0.001$, Student's $t$ test). (E) Representative images of comet assays of cortical neurons isolated from NTG and FUS-R521C mice. Quantification shows the percentage of cells with a comet tail (mean \pm SEM, unpaired Student's $t$ test). Scale bar: $50 \mu \mathrm{m}$. 

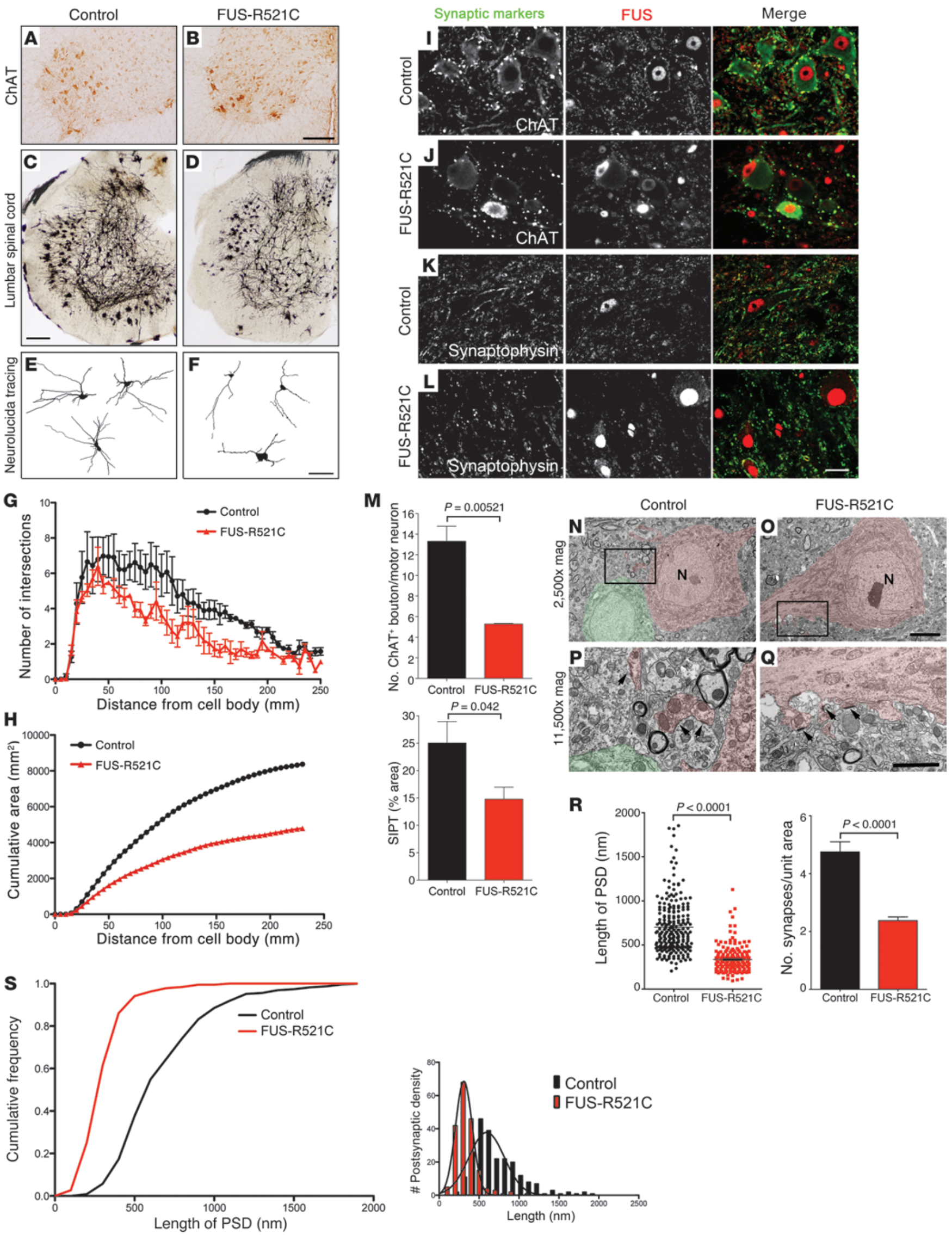


\begin{abstract}
Figure 4
Dendritic and synaptic defects in FUS-R521C spinal motor neurons. (A and B) ChAT immunostaining shows a modest reduction of motor neurons in cervical spinal cord of FUS-R521C mice (P37-P44). Scale bar: $100 \mu \mathrm{m}$. (C and D) Golgi staining shows reduced dendritic arborization in FUS-R521C motor neurons. Scale bar: $200 \mu \mathrm{m}$. (E and F) Neurolucida tracing of dendrites in control and FUS-R521C motor neurons. Scale bar: $100 \mu \mathrm{m}$. (G and $\mathbf{H}$ ) Sholl analyses show reduced dendritic intersections (from 50 to $250 \mu \mathrm{m}$ ) and reduced cumulative area of dendrites in FUS-R521C neurons. $P<0.0001$, 2-way repeated measures ANOVA. (I-M) Extensive colocalization of FUS with ChAT+ boutons and SIPT is reduced in FUS-R521C spinal cord. Student's $t$ test. Scale bar: $10 \mu \mathrm{m}$. (N-Q) EM images of control cervical spinal cord show synapses adjacent to 2 motor neurons (pink and green), many showing rosette-like pattern (arrows in P). In contrast, synapse density and size (arrows in $\mathbf{Q}$ ) are reduced in areas adjacent to FUSR521C motor neuron (pink). Scale bars: $5 \mu \mathrm{m}(\mathbf{0}) ; 2 \mu \mathrm{m}(\mathbf{Q})$. N, nucleus of spinal motor neurons. (R) Reduced PSD length and the density of synapse in FUS-R521C cervical spinal cord. $P<0.0001$, 2-way repeated measures ANOVA. (S) Reduced cumulative frequency and PSD size distribution in FUS-R521C neurons.
\end{abstract}

in the spinal cord of 2- to 3-month-old, age-matched control and FUS-R521C mice. In contrast to the elaborate dendrites in control spinal motor neurons, FUS-R521C motor neurons exhibited much more primitive dendritic branching (Figure 4, C and D). Neurolucida tracing showed that the dendritic arbors in control motor neurons had 6 to 8 intersections per radial distance within $100 \mu \mathrm{m}$ from the cell body, followed by a gradual reduction in the number of dendritic arbors from 100 to $250 \mu \mathrm{m}$ (Figure 4, E and $\mathrm{G})$. Compared with the control, the number of dendritic arbors in the FUS-R521C motor neurons showed no change within the first $50 \mu \mathrm{m}$ from the cell body, but a significant reduction was noted from 50 to $250 \mu \mathrm{m}$, resulting in reduced cumulative dendritic area (Figure 4, F-H). Similar dendrite phenotype in FUS-R521C motor neurons could be detected in FUS-R521C mice as early as P18 (Supplemental Figure 3, J and K). Since loss of motor neuron in the anterior horn of FUS-R521C mice was only about $20 \%$ at P16 (Supplemental Figure 3B), these results suggested that the dendritic defects in FUS-R521C motor neurons most likely preceded neuron loss and could contribute to spinal motor neuron degeneration in FUS-R521C mice.

To determine whether the dendritic phenotype in FUS-R521C spinal motor neurons affected synaptic connectivity, we used ChAT and synaptophysin immunostaining to characterize the density of synapses surrounding motor neurons (28). Consistent with the results in Figure 2, FUS proteins were present primarily in neuronal nuclei, but also showed extensive colocalization with $\mathrm{ChAT}^{+}$boutons and synaptophysin-immunoreactive presynaptic terminals (SIPT) (Figure 4, I and K). Remarkably, the density of $\mathrm{ChAT}^{+}$boutons and SIPT showed significant reductions in the anterior horn of FUS-R521C spinal cord (Figure 4, J, L, and M). To further characterize the synaptic defects, we used electron microscopy (EM) to ascertain the morphology and density of synapses within a $100 \mu \mathrm{m}$ radius of the cell body of spinal motor neurons. Consistent with the results from confocal microscopy (Figure 4, I-L), the cell bodies of control motor neurons were surrounded by synaptic terminals that were arranged as rosette-like structures (ref. 28 and Figure 4, N and P). In contrast, the size of postsynaptic density (PSD) and the number of synapses per unit area were reduced in FUS-R521C motor neurons (Figure 4, O and Q-S).
Dendritic and synaptic defects in the sensorimotor cortex of FUS-R521C mice. To investigate whether cortical neurons also exhibited similar defects, we examined the sensorimotor cortex in adult control and FUS-R521C mice using Nissl stain and layer-specific neuronal markers CTIP2 and FoxP1 and found no reduction in neuronal density in FUS-R521C mice (Supplemental Figure 4, A and B). Interestingly, Neurolucida tracings showed that the pyramidal neurons in layers IV-V of the sensorimotor cortex in FUS-R521C mice exhibited reduced dendritic complexity (Figure 5, A-D). Whereas the maximal length of the apical dendrites showed no detectable difference between control and FUS-R521C neurons, the number of dendritic branches (between 50 and $150 \mu \mathrm{m}$ from the cell body) and the cumulative dendritic area were significantly reduced in FUS-R521C neurons (Figure 5, E and F). Similar defects were also observed in the basal dendrites (Supplemental Figure 4, $\mathrm{C}$ and D). Furthermore, the total spine and mature spine densities on the apical and secondary dendrites of FUS-R521C neurons in sensorimotor cortex also showed significant reductions (Figure 5, G-K). Finally, EM analyses showed that the synaptic density, the length of the PSD, and the cumulative size frequency of PSD were all reduced in the sensorimotor cortex of FUS-R521C mice (Figure 5, L-P, and Supplemental Figure 4E).

$D N A$ damage and RNA splicing defects in Bdnf $5^{\prime}$ exons. To characterize the mechanism of dendritic and synaptic phenotype in FUSR521C neurons, we asked whether the increase in DNA damage in FUS-R521C neurons could contribute to these phenotypes. To this end, we used formamidopyrimidine-DNA glycosylase (FPG), a base excision repair enzyme, to identify oxidized purine residues in a number of neural genes that have been shown to regulate neuronal functions during DNA damage response and repair $(21,29)$. In this quantitative PCR-based (qPCR-based) assay, the relative abundance of oxidized DNA residues is reflected by the reduced yields in PCR products detected by locus-specific primers (Figure 6A). By screening a list of candidate genes with well-documented roles in dendritic growth and synaptic functions in the context of chromatin remodeling-dependent neurodegeneration (20), we found that the genomic DNA from the FUS-R521C brain showed a marked increase in oxidized purine residues in NR2A, GluR2, and 5' exons and 3'UTR of the Bdnf gene (Figure 6B). However, only the $B d n f$ gene showed consistent DNA damage in the brain and spinal cord. Given the important role of BDNF in dendritic growth and synapse formation, we decided to focus on its role in neurological phenotypes in FUS-R521C mice (30-32). Since the $5^{\prime}$ exons of $B d n f$ contain transcriptional start sites and the transcription of $B d n f$ involves splicing of the long intronic sequences (Figure 6C and refs. 33, 34), we asked whether FUS might also regulate the subsequent splicing of $5^{\prime}$ exons of $B d n f$ pre-mRNA (17). To test this, we performed qRT-PCR using RNA from control and FUS-R521C brains and primers that covered the $5^{\prime}$ splice donor sites from exons 1 to 6 (Figure 6C). Compared with control, RNA from FUS-R521C brain showed significant retention of the splice junctions for almost all 5' exons, especially exons 2, 4, and 6 (Figure $6 \mathrm{D})$. Interestingly, qRT-PCR using primers that detected the $3^{\prime}$ junction of intron 7 and exon 8 of $B d n f$ also detected a higher level of RNA transcripts that retained the $3^{\prime}$ splice acceptor site.

To determine if FUS-R521C mutant proteins might bind to the $5^{\prime}$ splice junctions of $B d n f$ pre-mRNA and interfere with its splicing, we performed CLIP using FUS antibodies to isolate the FUS-RNA complex, followed by qRT-PCR to determine how FUSR521C and WT FUS proteins differed in their ability to interact 

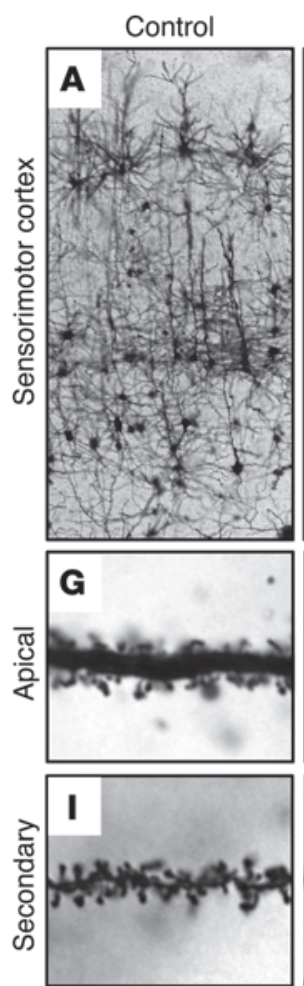

\section{$\mathbf{K}$}

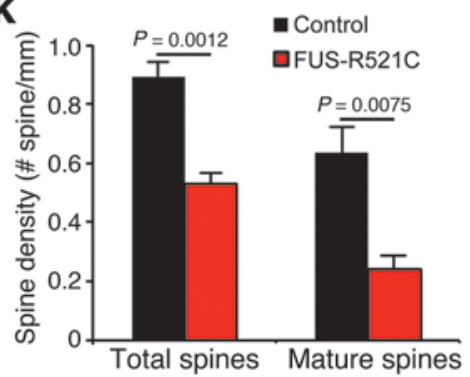

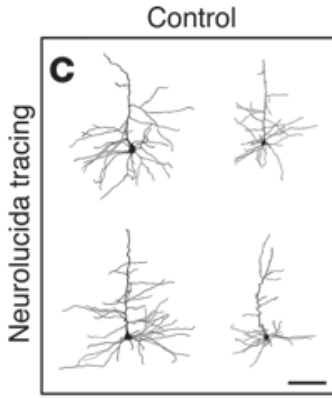
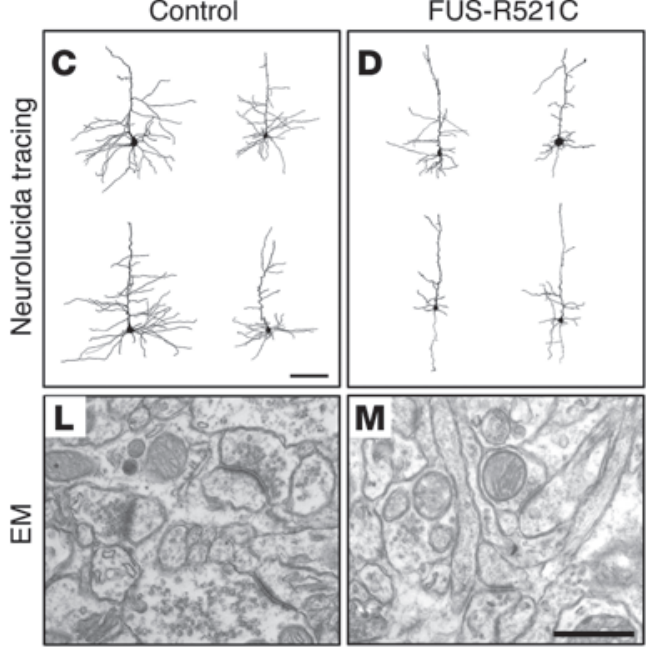

$\mathbf{N}$
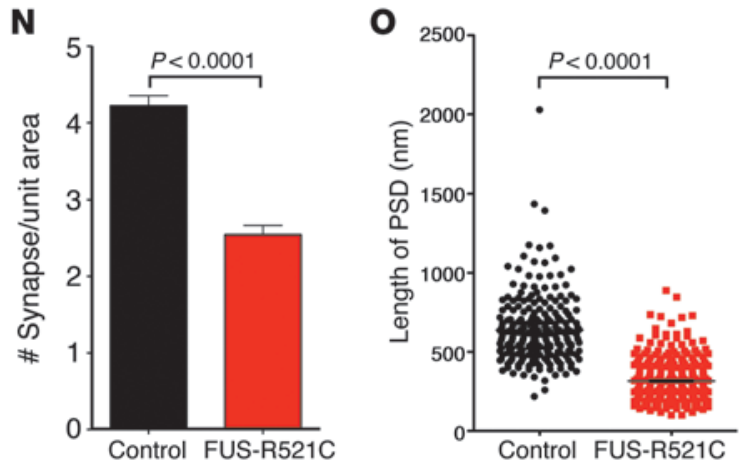

$\mathbf{P}$

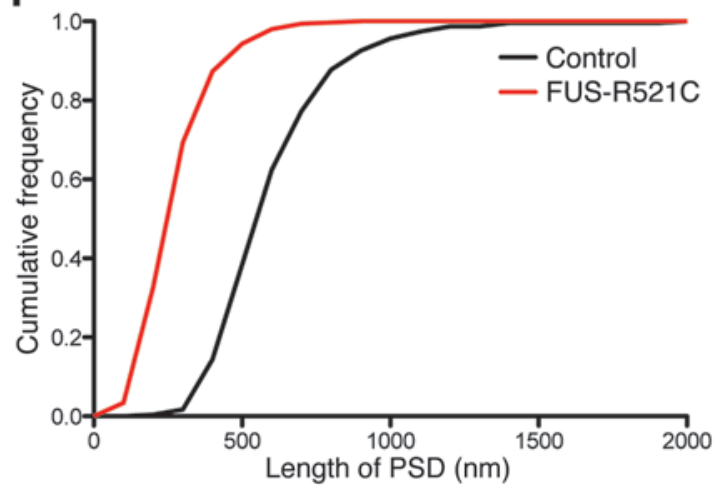

Apical dendrite

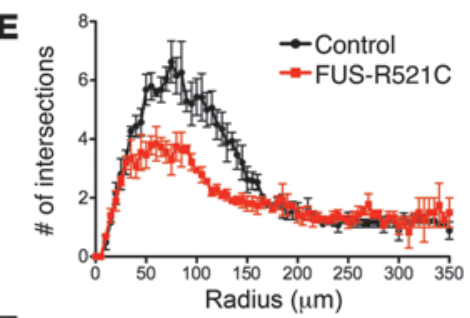

$\mathbf{F}$

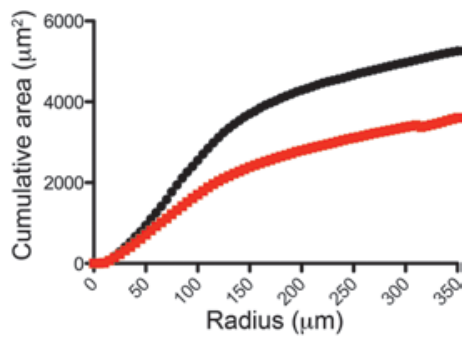

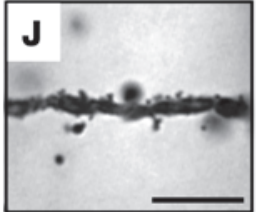

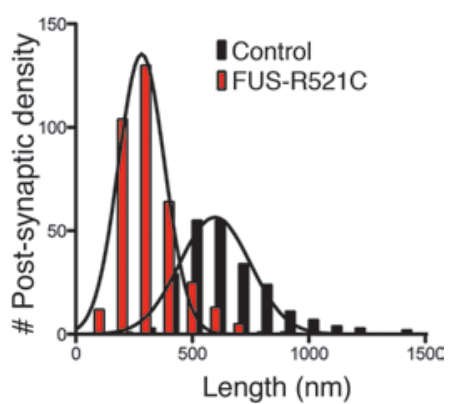

Figure 5

Dendritic and synaptic defects in neurons of the sensorimotor cortex in FUS-R521C mice. (A and B) Golgi staining shows reduced dendritic arborization in layers IV-V neurons in FUS-R521C sensorimotor cortex. Scale bar: $100 \mu \mathrm{m}$. (C and D) Neurolucida tracing of the apical and basal dendrites in control and FUS-R521C cortical neurons. Scale bar: $50 \mu \mathrm{m}$. (E and F) Reduced dendritic intersections in apical dendrite and cumulative areas of dendrites in FUS-R521C cortical neurons. $P<0.0001$, 2-way repeated measures ANOVA. (G-K) Reduced mature dendritic spine density in dendrites of FUS-R521C cortical neurons. Scale bar: $10 \mu \mathrm{m}$. Statistical analyses use 2-tailed Student's $t$ test, $P=0.001$ for total spine and $P=0.008$ for mature spine. (L-M) EM shows reduced synapse in the sensorimotor cortex of FUS-R521C mice. 2-tailed Student's $t$ test, $P<0.0001$. Scale bar: $1 \mu \mathrm{m}$. (N and $\mathbf{0}$ ) FUS-R521C cortex shows reduction in the length of the PSD and the number of synapse. $P<0.0001$, 2-way repeated measures ANOVA. (P) Reduced cumulative frequency and size of PSD in FUS-R521C cortex. Statistics for PSD length use 2-tailed Student's $t$ test, $P<0.0001$, and for cumulative frequency use 2-tailed Mann-Whitney test, $P=0.0042$.

with Bdnf pre-mRNA. Intriguingly, CLIP-qRT-PCR showed that in control brains, there was a modest binding of FUS to the 5' splice junction of exons 2, 4, and 6, but not exons 1, 3, and 5 (Figure $6 \mathrm{E})$. In contrast, FUS proteins in the FUS-R521C brains exhibited a marked increase in binding to the $5^{\prime}$ splice junction for exon 2 .
Similar trends were observed in exons 4 and 6, though the differences were not statistically significant.

FUS-R521C forms more stable complexes with BdnfRNA. To further characterize the interactions between FUS and $B d n f$ pre-mRNA, we used EMSA to examine the interactions between recombinant WT 
FUS proteins (GST-FUS-WT) or FUS-R521C proteins (GST-FUSR521C) and a series of oligoribonucleotides (RNA oligos) that covered the $5^{\prime}$ splice junction from exons 1 to 7 (Figure 6C). This approach showed that GST-FUS-WT could indeed form a complex with RNA oligos from the 5 ' splice junctions of exons 2 , 4, and 6 , but not with those from $1,3,5$, or 7 (Figure 7A). Interestingly, GST-FUS-R521C proteins also interacted with the same RNA oligos, but the GST-FUS-R521C-RNA complexes tended to migrate at higher molecular mass ranges (Figure 7A). Since FUS has been implicated in the transport of RNA granules in dendrites (35), we asked whether FUS might interact with the $3^{\prime}$ UTR of $B d n f$ mRNA, which is required for transport along dendrites (30). Survey of the $B d n f$ long $3^{\prime}$ UTR sequences identified 4 regions that contained potential the FUS-binding motif, GGUG (Bdnf3'UTR\#1 to \#4, Figure $6 \mathrm{C}$ and ref. 36). Interestingly, only RNA oligo Bdnf $33^{\prime} \mathrm{UTR} \# 2$ interacted with FUS. Similar to the results in Figure 6A, GST-FUSR521C proteins also formed high molecular RNA-protein complexes with Bdnf 3' UTR\#2 (Figure 7B).

To test whether FUS-R521C proteins might form a more stable protein-RNA complex than WT proteins, we performed competition assays by increasing the concentrations of nonradioactive RNA oligos to displace GST-FUS-WT or GST-FUS-R521C from its complex with radioactively labeled $B d n f 3^{\prime} \mathrm{UTR} \# 2$ (Figure 7C). Our results indicated that nonradioactive RNA oligo could displace GST-FUS-WT from the protein-RNA complex with an $\mathrm{IC}_{50}$ of $0.73 \mu \mathrm{M}$. In contrast, the GST-FUS-R521C-RNA complex was more stable with an $\mathrm{IC}_{50}$ of $2.19 \mu \mathrm{M}$ (Figure $7, \mathrm{C}$ and D). Even with a $10 \mu \mathrm{M}$ nonradioactive probe (400-fold higher in concentration), more than $20 \%$ of the radioactive GST-FUS-R521C-RNA complex still remained bound.

Reduced Bdnf mRNA impairs TrkB signaling in FUS-R521C mice. To determine how FUS-R521C affected the overall Bdnf mRNA levels in neurons, we used in situ hybridization probes that detected either the Bdnf coding region (BdnfCDS) or $3^{\prime}$ UTR ( $B d n f 3^{\prime}$ UTR $[\mathrm{L}])$ and showed that $B \operatorname{dnf} \mathrm{mRNA}$ was quite abundant in the cytoplasm and dendrites of control spinal motor neurons and cortical neurons (Figure 8A). In contrast, $B d n f$ mRNA was significantly reduced in the cell body and dendrites of neurons in the sensorimotor cortex and in spinal motor neurons (Figure 8A). Similar results were observed in cultured cortical neurons from FUS-R521C mice (Supplemental Figure 5). To determine the level of BDNF proteins, we performed immunoprecipitation followed by Western blot and showed that the amount of pro-BDNF and mature BDNF proteins in the brain and spinal cord tissue was significantly lower in FUSR521C mice (Figure 8, B and C, and ref. 37). Consistent with these results, activated TrkB receptors in FUS-R521C mice, detected using a phospho-TrkB ( $\mathrm{p}$-TrkB) antibody (38), were reduced to $30 \%$ and $12 \%$ of the levels in control brain and spinal cord, respectively, whereas total TrkB proteins remained unchanged (Figure 8, B and D). Interestingly, the reduction of BDNF protein levels and TrkB activation in FUS-R521C mice was not detected on P2, but became progressively worsened at P16 and P60 (Supplemental Figure 6). In contrast, activation of another neurotrophic receptor c-Ret appeared unaffected in the spinal cord of FUS-R521C mice at the same postnatal stages (Supplemental Figure 6).

Given BDNF's role in promoting dendritic growth and synaptic functions $(31,32)$, we asked whether exogenous BDNF might restore TrkB signaling in FUS-R521C-expressing neurons. To test this, we cultured E17.5 cortical neurons from control and FUSR521C embryos for 14 DIV, treated them with or without BDNF (10 $\mathrm{ng} / \mathrm{ml}$ ), and then collected them for Western blot analyses. Similar to the results in Figure 8, A-D, FUS-R521C cortical neurons showed much lower levels of $\mathrm{p}$-TrkB than WT neurons in the absence of BDNF (Figure 8, E and F). Following treatment with BDNF, however, both WT and FUS-R521C neurons showed comparable levels of p-TrkB. To further determine whether exogenous BDNF could ameliorate the dendrite phenotype in FUS-R521C-expressing neurons, we transfected WT cortical neurons at 7 DIV with GFP, GFP-FUSWT, or GFP-FUS-R521C plasmids and characterized the effects of FUS on the dendritic growth. Whereas the control GFP neurons showed a progressive increase in dendritic branches and total length from day 1 to 7 after transfection (Figure 8, G and H), neurons expressing GFP-FUS-WT proteins showed very limited growth of dendrites, consistent with the report that overexpression of WT FUS proteins could also cause neurodegeneration (39). Remarkably, neurons expressing FUS-R521C proteins showed retraction in dendritic growth, with a marked reduction in branch points and total length (Figure 8, G and H, and Supplemental Figure 7). Interestingly, adding exogenous BDNF (10 ng/ml) partially ameliorated the negative effects of WT FUS and FUS-R521C proteins on dendritic growth in cortical neurons (Figure 8, G and I). Using automated microscopy, we showed that cortical neurons expressing WT or mutant FUS proteins had a very modest effect on neuronal survival (Supplemental Figure 8), supporting the idea that the effects of FUS-R521C proteins on dendritic growth were likely not due to cell death.

$R N A$-seq transcriptome analyses reveal transcriptional and splicing defects in FUS-R521C mice. Our results showing that exogenous BDNF only partially rescued dendrite phenotype in FUS-R521C cortical neurons suggested that FUS-R521C may control the transcription and/or splicing of additional target genes critical for dendritic growth and synaptic functions. To test this, we performed RNA-seq analyses on paired-end Illumina libraries from the spinal cords of 3 P38 disease end-stage FUS-R521C mice and 3 age-matched controls. A total of $37.33 \pm 3.77 \times 10^{6}$ reads per sample were obtained, and more than $98 \%$ of the reads were uniquely mapped to the mouse genome. We first analyzed the RNA-seq data using DESeq, which interrogated high throughput sequencing data for differential expression (40). Compared with controls, 766 genes in the FUS-R521C spinal cord showed significant differential expression (Figure 9A and Supplemental Table 1). Functional annotations of all 766 genes using DAVID Bioinformatics 6.7 revealed more than 10 Gene Ontology (GO) groups that were implicated in pattern formation (paralogs of the Hox10, Hox11, and Hox13 that regulate motor neuron specification and muscle innervation) (41, 42), extracellular matrix, neuron projection, sterol biosynthesis, immune-mediated processes, ion channels, and synaptic functions (Figure 9B and Supplemental Table 1). Further analyses showed that genes with increased reads consisted of 3 major GO categories, including pattern formation, extracellular matrix, and immunemediated responses, e.g., chemotaxis and phagocytosis (Figure 9A, inset, and Supplemental Table 1), whereas genes with reduced reads were mainly related to synaptic functions, ion channel activity, neuron projection and sterol biosynthesis (Supplemental Table 1). Remarkably, many genes in the extracellular matrix assembly category, including GO;0005581, GO:0005201, GO:0005578, and GO:0031012, were identified as the transcriptional targets of DNA damage response gene Cockayne syndrome B (CSB) and HDAC1 (43). Together, these results provided further molecular insights that potentially connect DNA damage and the profound dendritic/ synaptic defects in FUS-R521C mice. 
A Oxidized purine residues
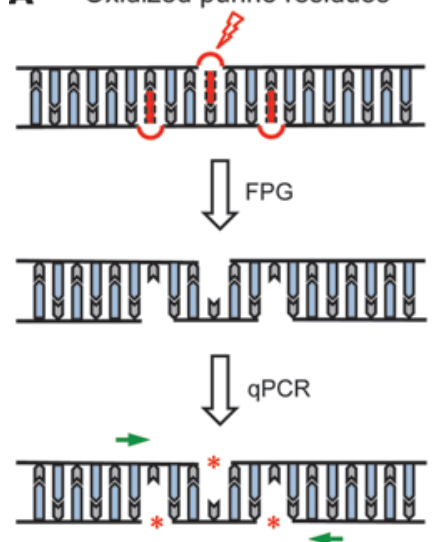

B
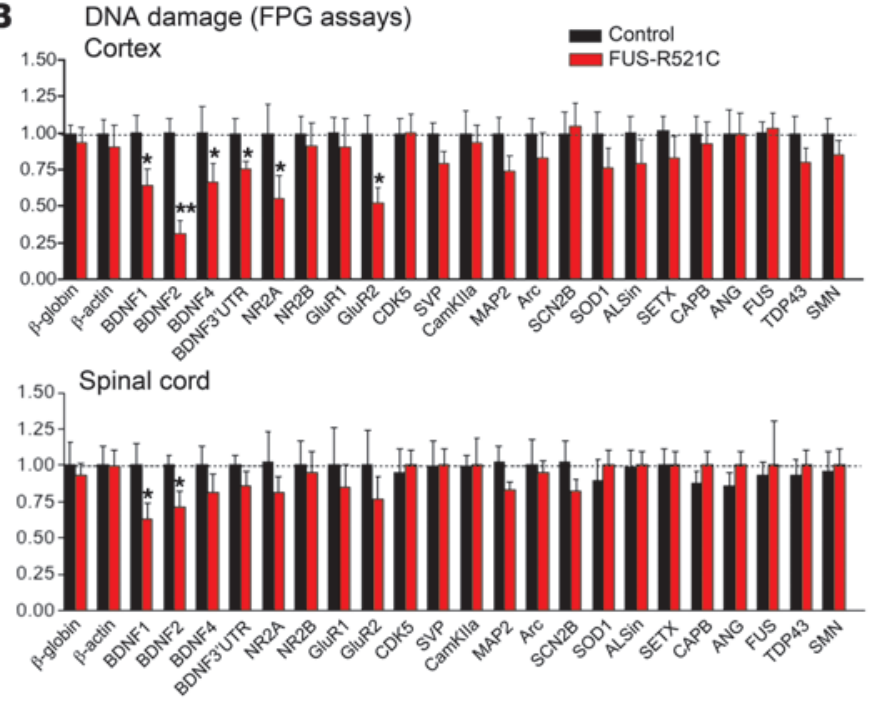
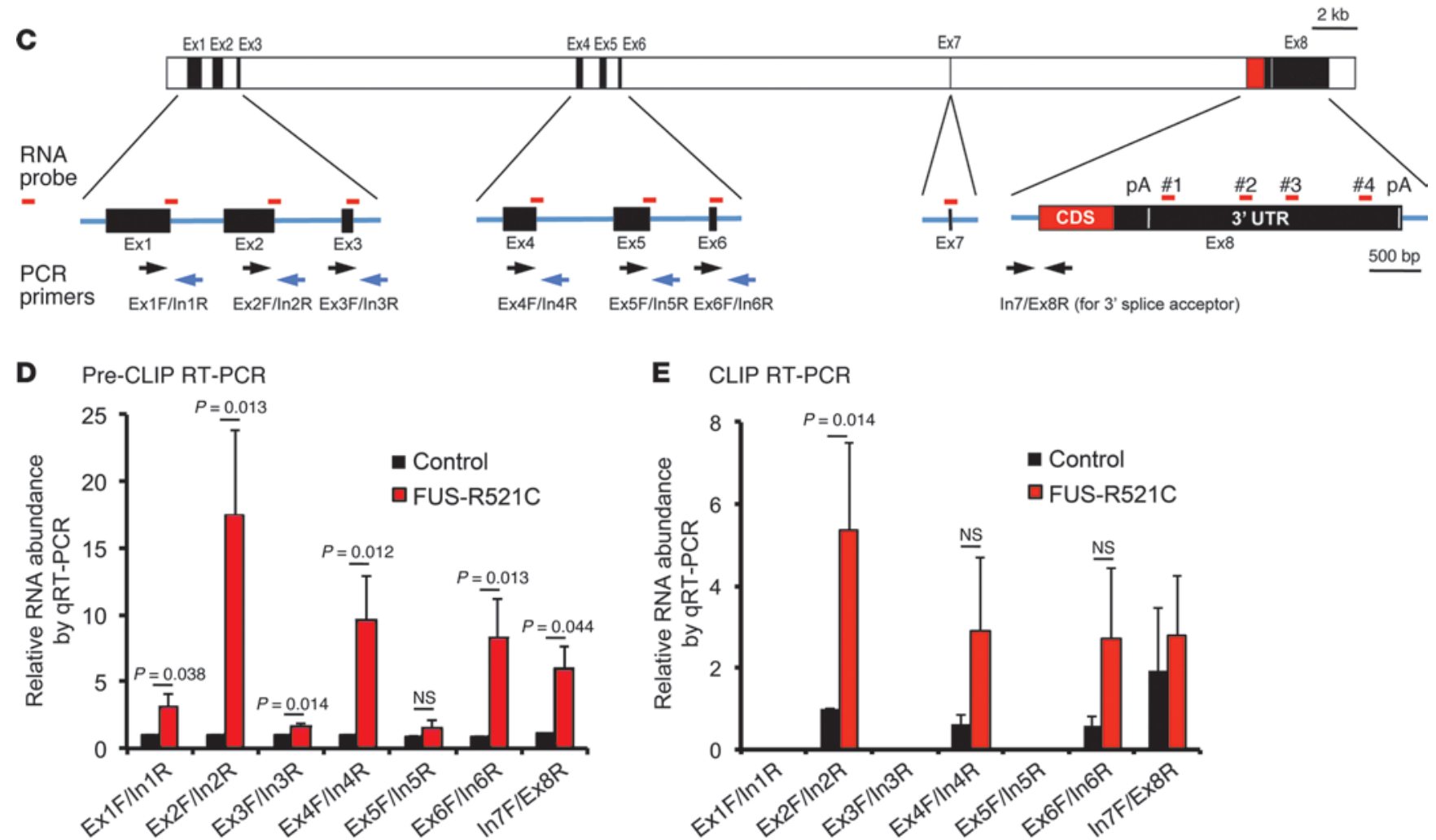

E CLIP RT-PCR

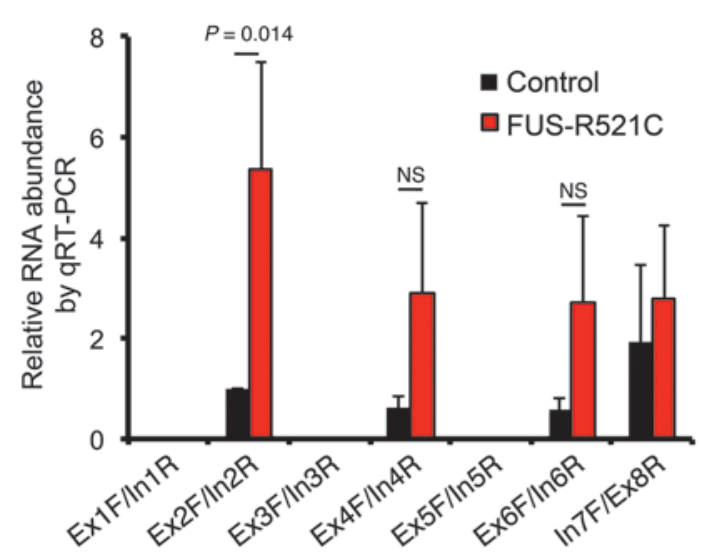

Figure 6

FUS-R521C mice show increased DNA damage and splicing defects in $5^{\prime}$ noncoding exons in the Bdnf gene. (A) Schematic diagrams of the qPCR-based FPG assay to identify oxidized (or "damaged") nucleotides in genomic DNA. (B) FPG assays reveal DNA damage in the 5' noncoding exons of the Bdnf gene in cortex and spinal cord in FUS-R521C mice. Statistics use Student's $t$ test, ${ }^{\star} P<0.05 ;{ }^{* \star} P<0.01$. (C) A schematic diagram of the mouse Bdnf gene, which includes seven 5' noncoding exons and exon 8, which contains the entire coding sequence and 3' UTR. PCR primers to detect the retention of $5^{\prime}$ splice junction are highlighted as black arrows (forward) and blue arrows (reverse). Red bars indicate the synthetic oligoribonucleotides that contain the $5^{\prime}$ splice junctions in noncoding exons. (D) qRT-PCR assays detect the marked retention of $5^{\prime}$ splice junction sequences in the Bdnf mRNA in FUS-R521C brain. (E) Crosslinking immunoprecipitation (CLIP) qRT-PCR assays show that more FUS proteins are bound to the $5^{\prime}$ splice junction sequences in exons 2, 4, and 6 in the Bdnf mRNA in the brain of FUS-R521C mice. Statistics in $\mathbf{D}$ and $\mathbf{E}$ use Student's $t$ test. 
A

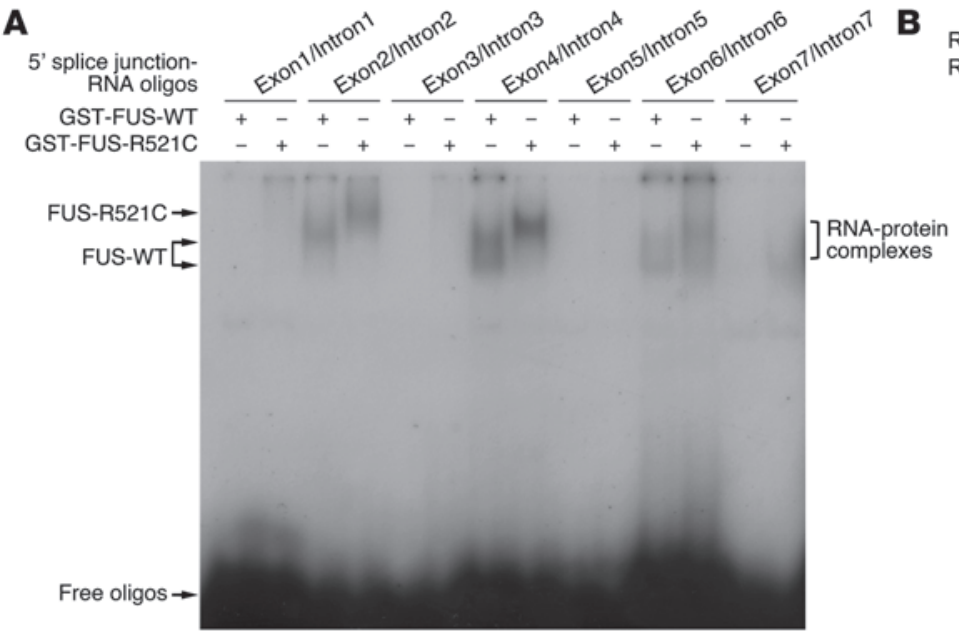

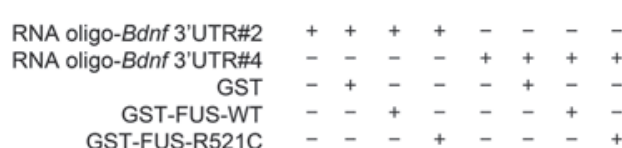
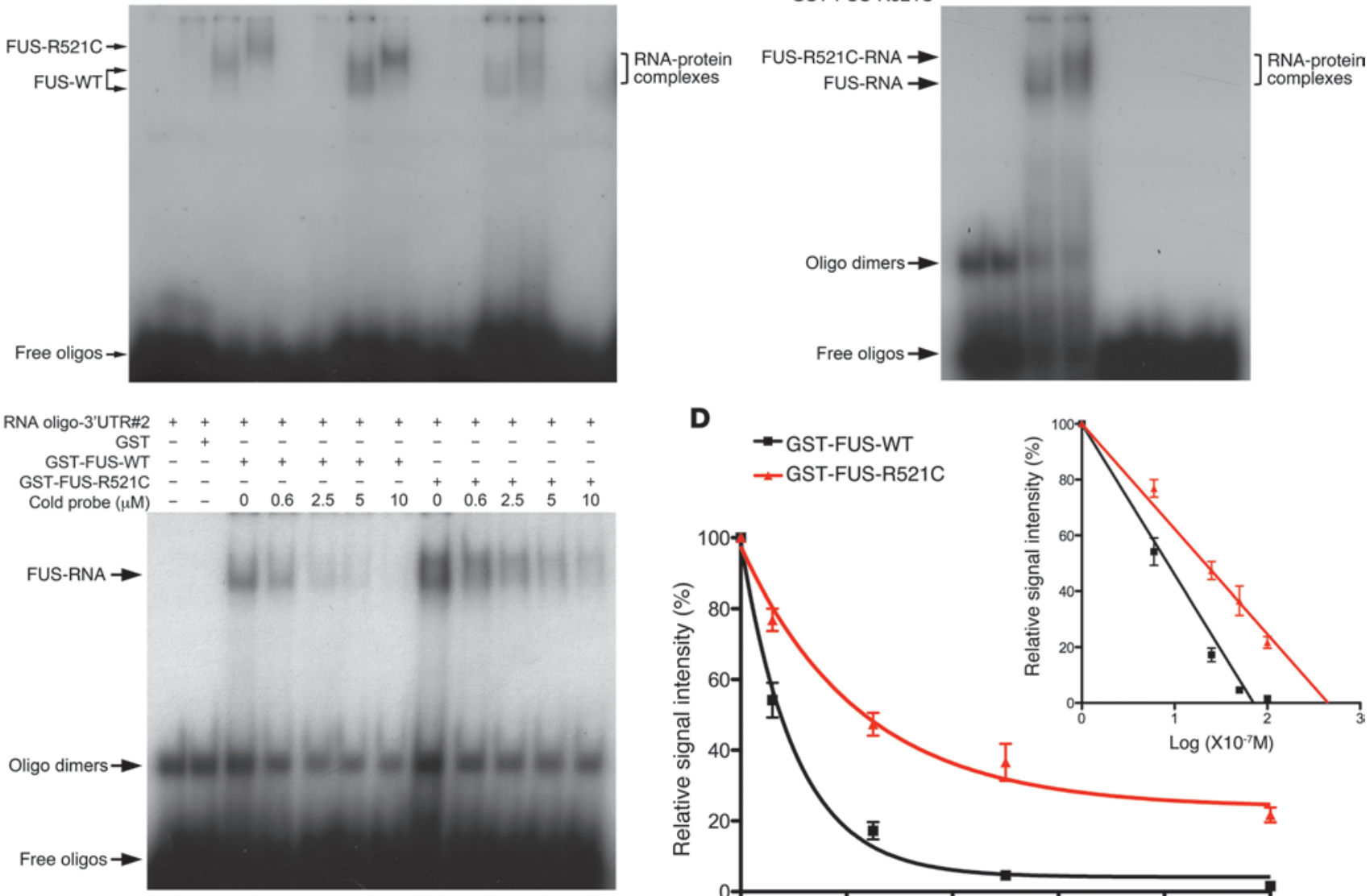

D
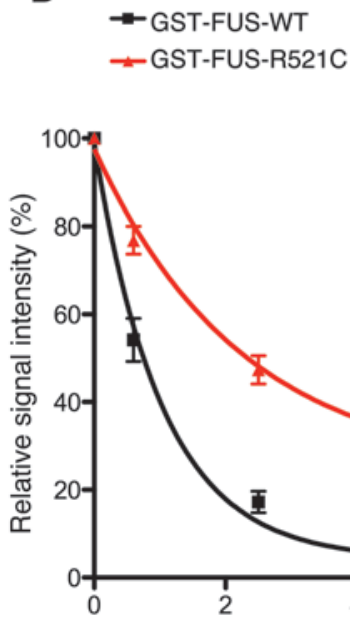

GST-FUS-R521C
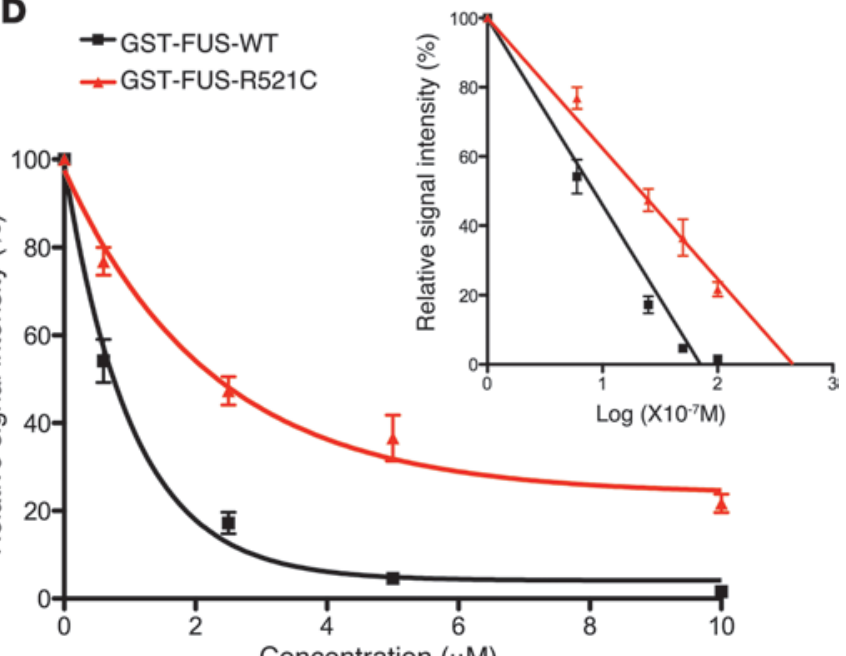

\section{Figure 7}

FUS-R521C proteins form more stable complexes with Bdnf RNA and reduce splicing efficiency. (A) EMSAs show both recombinant GST-WT FUS (GST-FUS-WT) and GST-FUS-R521C proteins form complexes with radioactive RNA oligos that contain sequences from the 5' splice junction of Bdnf exons 2, 4, and 6. Compared with GST-FUS-WT, the RNA-protein complexes formed by GST-FUS-R521C tend to show slower migration, suggesting a higher molecular weight complex or more stable complex formation. (B) Both GST-FUS-WT and GST-FUS-R521C proteins also interact with RNA oligo-Bdnf-3' UTR\#2, but not Bdnf-3' UTR\#4. (C and D) Competition assays show that nonradioactive ("cold") RNA oligos can displace WT FUS from the RNA-protein complex at the $\mathrm{IC}_{50}$ of $0.73 \mu \mathrm{M}$. In contrast, the GST-FUS-R521C-RNA complex was more stable and required higher concentration of cold probe to dissociate RNA-protein complexes $\left(\mathrm{IC}_{50}=2.19 \mu \mathrm{M}\right)$.

To characterize the splicing defects in FUS-R521C mice, we used SpliceMap to generate the Relative Intron retention Index (RII) from the control and FUS-R521C spinal cord RNA-seq data sets (44). This analysis showed that 1,056 events were retained at higher levels in the FUS-R521C samples, whereas 758 events were retained at higher levels in the WT $(P<0.05)$ (Figure 9C). Furthermore, compared with the 192,168 introns in Reference Sequence (RefSeq) data, the events identified in the FUS-R521C samples showed almost identical size distribution, with no preference toward small or large introns (Figure 9D). To determine whether the alternative splicing pattern was affected by FUS-R521C mutation, we calculated the relative junction index (RJI) in the spinal cord RNA-seq data sets from WT and FUS-R521C mice. These analyses identified 1,246 introns that were retained at higher levels in FUS-R521C samples $(P<0.05)$, with 37 introns with RJI ratio greater than 2 . In contrast, only 177 introns were retained at higher levels in the WT samples $(P<0.05)$, including 11 with RJI ratios greater than 2 .

The GO analyses from DESeq showed increased reads in many extracellular matrix genes, including several collagen and cadherin genes (Supplemental Table 1). Due to the roles of these genes in neurite outgrowth and synaptic specificity $(45,46)$, we reasoned that FUS-R521C might also cause defects in the splicing and exon selection in these genes. Indeed, Col7a1 RNA in the FUS-R521C spinal cord showed a marked increase of reads in DESeq (4.35fold increase, adjusted $\left.P<2.58 \times 10^{-06}\right)$, and RNA-seq data from the FUS-R521C spinal cord showed increased use of exons and intron retention in Col7a1 RNA (Figure 9, E and F, red brackets and arrows). The RNA-seq results on Col7a1 were confirmed by qRT-PCR using primers that detected $5^{\prime}$ or $3^{\prime}$ splice junctions. 
A

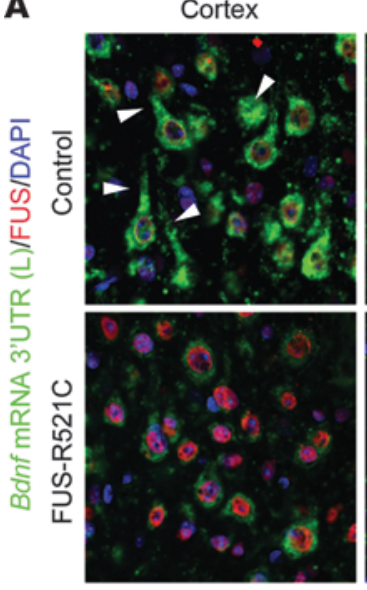

D

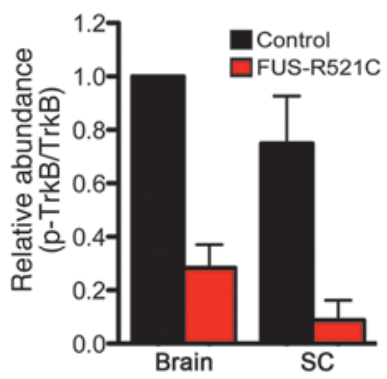

Spinal cord

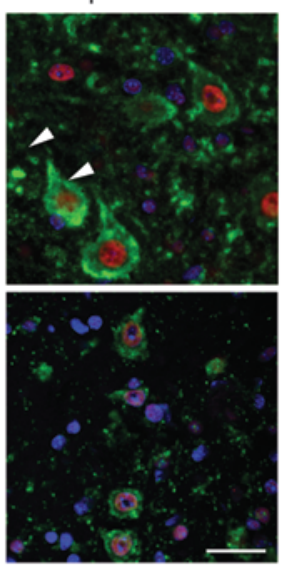

B

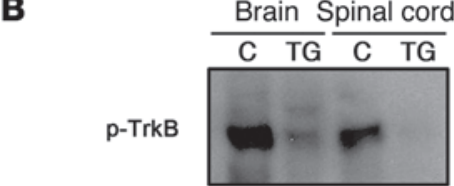

Full-length TrkB

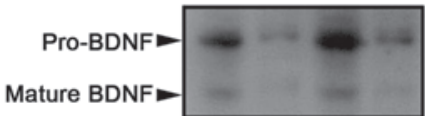

Actin
E

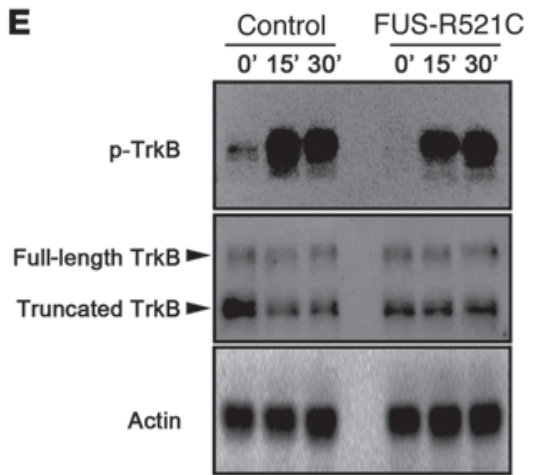

C

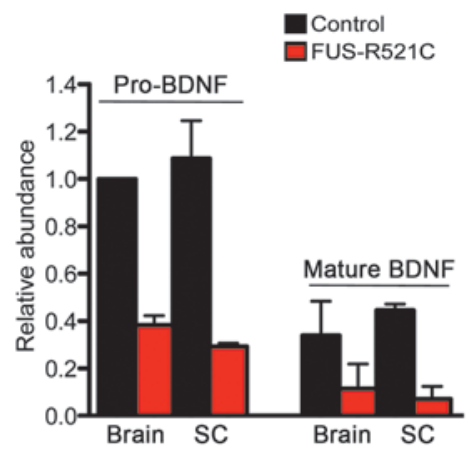

\section{F}

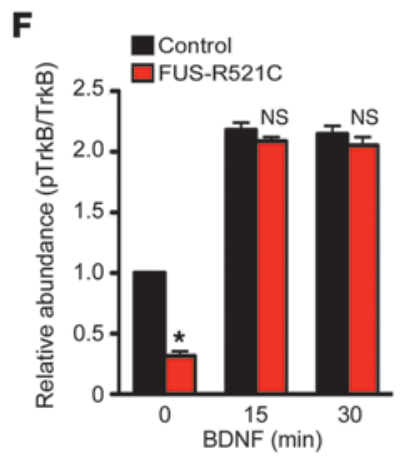

G Basal condition
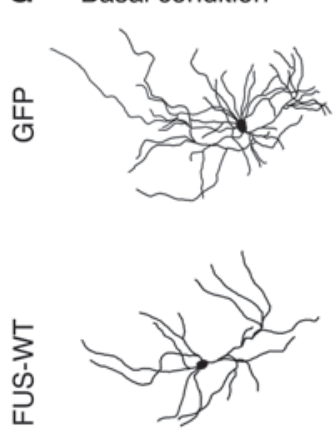

एู
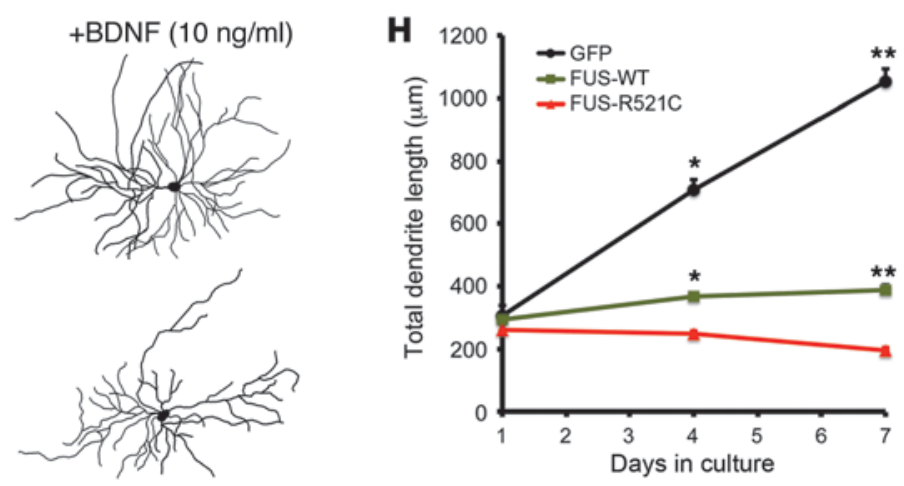

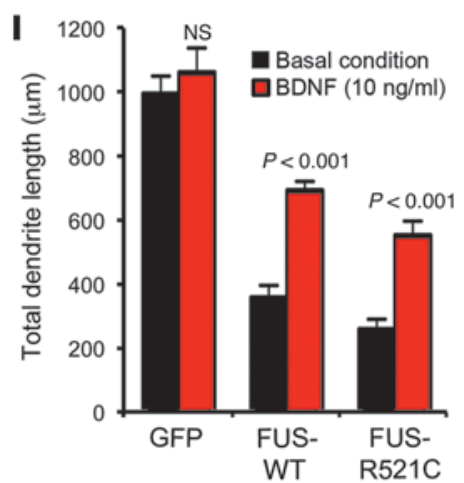

Figure 8

BDNF restores TrkB activation and partially ameliorates dendrite phenotype in cortical neurons expressing FUS-R521C. (A) In situ hybridization shows reduced Bdnf mRNA in cortical and spinal motor neurons in FUS-R521C mice. Bdnf mRNA can be detected in the cell body and dendrites of control neurons (arrowheads), but is significantly reduced in FUS-R521C neurons. Scale bar: $20 \mu \mathrm{m}$. (B-D) Western blots and quantification showing reduced pro-BDNF, mature BDNF, and TrkB phosphorylation in FUS-R521C brain and spinal cord, without affecting total TrkB protein levels. (E and F) BDNF treatment $(10 \mathrm{ng} / \mathrm{ml})$ restores TrkB activation in cortical neurons from FUS-R521C embryos. ${ }^{*} P<0.01$. (G) Cultured neurons expressing WT FUS or FUS-R521C show reduced dendritic growth from DIV1 to DIV7, which can be partially ameliorated by exogenous BDNF $(10 \mathrm{ng} / \mathrm{ml})$. Scale bar: $20 \mu \mathrm{m}$. (H) Quantification of total dendrite length in control neurons and neurons expressing WT FUS or FUS-R521C from DIV1 to DIV7. ${ }^{*} P<0.05 ;{ }^{* \star} P<0.001$. (I) BDNF treatment partially restores dendritic growth in neurons expressing FUS or FUS-R521C. Statistics in $\mathbf{C}, \mathbf{D}, \mathbf{F}, \mathbf{H}$, and I use 2-tailed Student's $t$ test, $n=3$. 
Similarly, RNA-seq also showed increased RII and RJI indices in Col24a1, Col4a1, Col4a2, and Col4a5 genes (Supplemental Figure 9 and Supplemental Table $1, P<0.05$ ), which have been shown to regulate target innervation via the Agrin and Slit-Robo mechanisms $(47,48)$. In contrast, splicing of Col15a1, Col16a1, and Mapt (microtubule-associated protein or tau) was not affected (Supplemental Figure 9).

\section{Discussion}

In this study, we provide evidence that transgenic mice expressing a common FALS-associated FUS mutation, FUS-R521C, develop early postnatal lethality with motor behavioral deficits due to profound dendritic and synaptic phenotypes in the spinal motor neurons and cortical neurons. Consistent with our recent findings in FALS patients with the same mutation (7), FUS-R521C transgenic mice also show a robust increase in DNA damage repair defects in cortical and spinal motor neurons. By screening a number of neural genes that have been implicated in neurodegeneration, we show that increase of DNA damage can be identified in the $5^{\prime}$ exons of the $B d n f$ gene. Furthermore, we provide evidence that the aberrant property of FUS-R521C to form more stable proteinprotein and protein-RNA complexes could alter the equilibrium of FUS-RNA interactions and lead to a severe impairment in BDNFTrkB signaling. Finally, to gain more insights into the mechanisms of FUS-R521C phenotypes, we performed RNA-seq transcriptome analyses in the spinal cord of FUS-R521C mice and further revealed defects in the transcription and RNA splicing. Together, these results collectively support a model in which mutant FUS$\mathrm{R} 521 \mathrm{C}$ proteins acquire abnormal gain-of-function properties in protein-protein and protein-RNA complex formation to dominantly inhibit the normal physiological functions of FUS in DNA damage response/repair and RNA splicing of neural genes critical for dendritic growth and synaptic formation (Figure 10).

Several recent studies indicate that recombinant WT and mutant FUS proteins tend to form filamentous aggregates (23, 49). However, it remains unclear how FUS mutations might perturb biological processes, such as RNA splicing and transport. Our results indicate that mutant FUS-R521C proteins indeed have abnormal gain-of-function properties in protein-protein and protein-RNA interactions that contribute to the severe defects in dendritic growth and synaptic functions. For instance, in protein lysates from the brains and spinal cords of FUS-R521C transgenic mice and HEK293T cells coexpressing WT FUS and FUS-R521C, FUS-R521C mutant proteins consistently showed a higher propensity to form protein complexes with WT FUS proteins (Figure 2). Since FUS mutations are transmitted in an autosomal dominant fashion, it is conceivable that the constitutive, more stable interaction between FUS-R521C and WT FUS may have dominant negative effects that could interfere with normal functions of WT FUS proteins. In addition, this abnormal gain-of-function property in FUS-R521C may have a negative impact on the recruitment of other FUS-interacting cofactors, such as chromatin remodeling factor HDAC1, which is normally recruited to DNA damage foci (Figures 3 and 10; ref. 7). Aside from the abnormal properties of FUS-R521C in its interaction with FUS and HDAC1, our results show that FUS-R521C mutant proteins can also form more stable protein-RNA interactions, which perturbs $B d n f$ pre-mRNA splicing by interfering with the equilibrium, kinetics, and perhaps the stoichiometry of interaction with RNA (Figures 6, 7, and 10; ref. 50). In agreement with our results, another FALS-related muta- tion, FUS-R521G, when expressed in HEK293 cells, causes a drastic shift in binding preference from the intronic sequences to $3^{\prime}$ UTR sequences (18). Based on these results, it is possible that the more stable interactions between mutant FUS-R521C proteins and the $3^{\prime}$ UTR of $B d n f$ mRNA may also interfere with its transport to distal dendrites where local protein synthesis can further regulate dendritic growth and synaptic functions (Figure 8 and Supplemental Figure 5). Given the critical role of $3^{\prime}$ UTR in $B d n f$ mRNA in dendrite morphogenesis and energy homeostasis of hypothalamic neurons $(30,51)$, it is plausible that the gain-of-function properties of FUS-R521C mutant proteins in binding with the 3' UTR of $B d n f$ mRNA may also interfere with these important functions.

Several lines of evidence indicate that effects of FUS-R521C on the splicing of $B d n f$ mRNA are probably not due to secondary effects of general pathology in the transgenic mice. First, the defects in BDNF production in FUS-R521C mice appear to develop in an age-dependent manner (Supplemental Figure 6), and exogenous BDNF can effectively restore TrkB activation in FUS-R521C neurons (Figure 8). Second, in contrast to the severe reduction in $\mathrm{p}$-TrkB levels, activation of neurotrophic factor GDNF receptor c-Ret shows no detectable reduction (Supplemental Figure 6). Finally, the BDNF levels and TrkB activation show no detectable reduction in the spinal cord of SOD1G93A transgenic mice at end-stage (Supplemental Figure 10). These results support the idea that the molecular defects in FUS-R521C mice develop progressively in postnatal life, perhaps due to the prolonged accumulation of abnormal protein-protein and/or protein-RNA complexes initiated by mutant FUS proteins.

Previous studies have shown that WT FUS proteins are required for DNA damage repair $(5,6)$. In our recent study (11), we show that the interaction between WT FUS and HDAC1 plays an essential role in DNA damage response and repair in neurons. Unlike WT FUS, many FALS-associated FUS mutations show severe deficiencies in this process (7). These results raise the intriguing question as to whether FUS mutations may cause widespread DNA damage or more selective defects in certain genetic loci? To address this question, we use PCR-based FPG assays to screen for evidence of DNA damage in a number of genes in which chromatin remodeling defects have been associated with neurodegeneration (20). Our results show that DNA damage is not widespread in FUS-R521C mice, but can be detected in $5^{\prime}$ noncoding exons of the $B d n f$ gene in both spinal cord and brain (Figure 6). While the exact mechanism or mechanisms leading to DNA damage in selective genetic loci in FUS-R521C mice remain unclear, these results suggest that DNA damage in these mice may not be random and that epigenetic modifications and the state of chromatin may influence the specificity of DNA damage in selective genetic loci $(20,52)$.

The DNA damage repair mechanism encompasses molecular machinery that regulates chromatin remodeling, transcriptional control, and RNA splicing (9-11). Perturbations to these processes lead to serious consequences, including abnormal cell growth, apoptosis, and neurodegeneration (53). Although our results identify DNA damage and RNA splicing defects in Bdnf as potential contributing factors to the dendritic and synaptic phenotypes in FUS-R521C mice, exogenous BDNF can only partially rescue the dendritic defects in FUS-R521C-expressing neurons, despite its ability to activate $\mathrm{TrkB}$ receptors in these neurons (Figure 8). These results argue that the additional functions of FUS in transcription and RNA processing may further contribute to the profound dendritic and synaptic defects in FUS-R521C mice. 
A

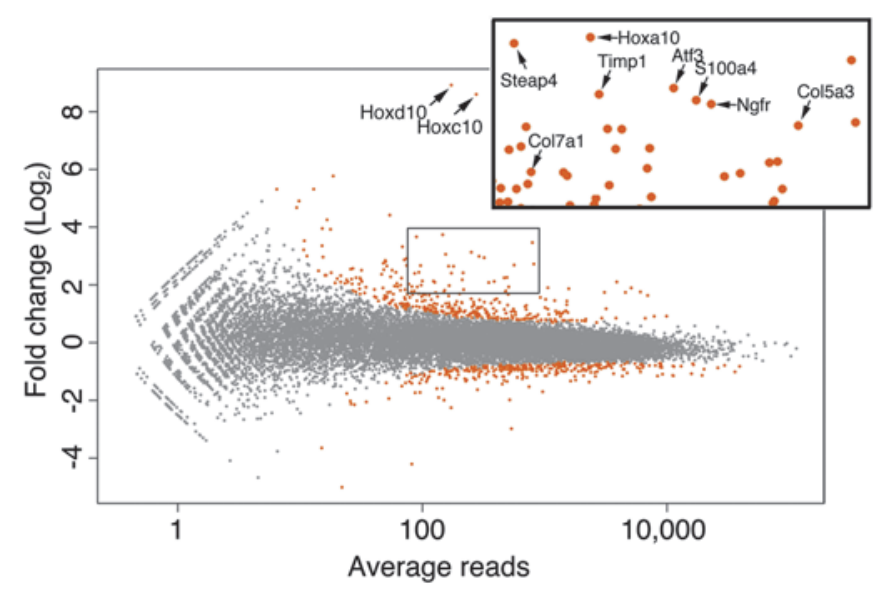

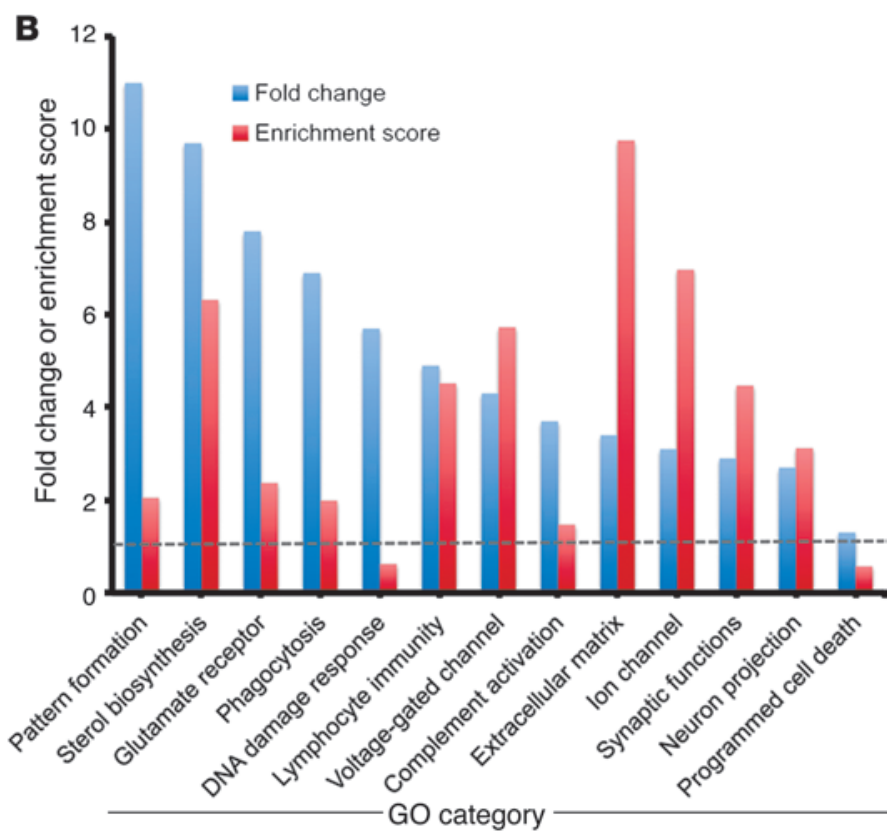

C

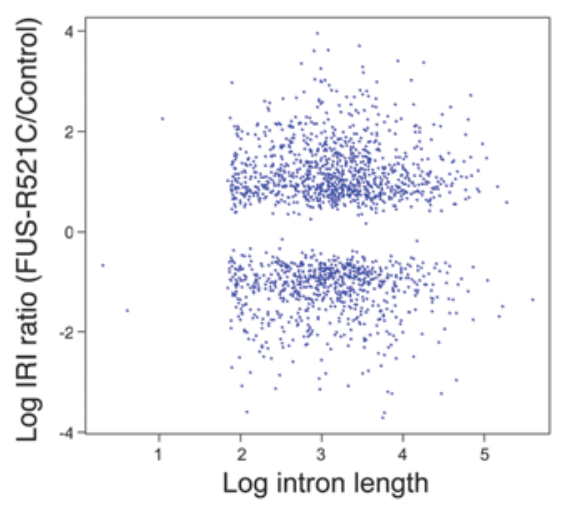

D

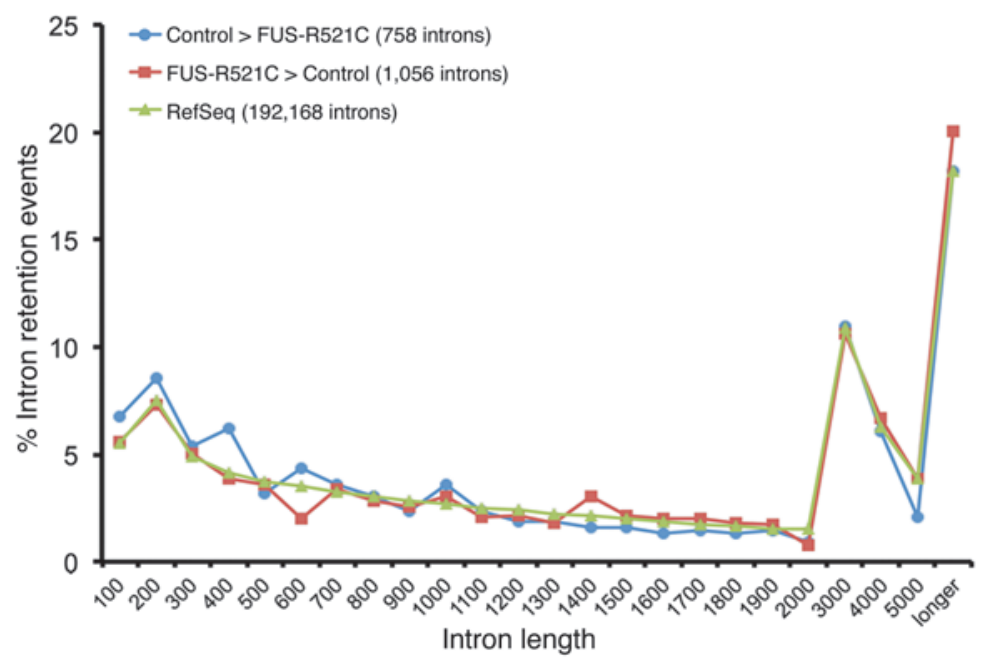

E

Scale

Chr. 9

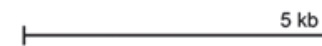

$5 \mathrm{~kb}$

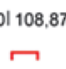
$\square$<smiles>C1C[C@H]2CCC[C@@H]2C1</smiles>
W hllt .

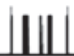

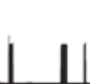

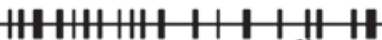

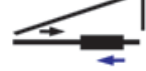

In107F/Ex108R

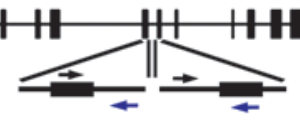

Ex112F/In112R In112F/Ex113R
F

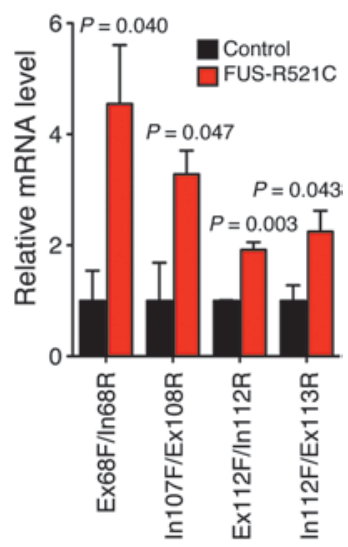




\section{Figure 9}

RNA-seq analyses reveal transcription and splicing defects in FUSR521C spinal cord. (A) DESeq analyses of RNA-seq data reveal 766 differentially expressed genes in FUS-R521C spinal cord. All mice were euthanized at P38, with the FUS-R521C mice reaching disease endstage. (B) Fold change and enrichment scores of the GO terms from DAVID Bioinformatics analyses of the differentially expressed genes in FUS-R521C spinal cord. (C) Scattered plot generated by SpliceMap demonstrates intron retention events and the log ratio of intron retention index (RII) of FUS-R521C/WT ( $y$ axis) plotted against the log of the intron length (bp) ( $x$-axis). (D) Significantly retained introns in the spinal cord of FUS-R521C mice and RefSeq introns are grouped by length and plotted as a percentage of the total introns. ( $E$ and $\mathbf{F})$ The mouse Col7a1 gene encompasses $54 \mathrm{~kb}$ on chromosome 9 and contains 119 exons. In FUS-R521C spinal cord, Col7a1 mRNA shows evidence of excessive inclusion of cassette exons (bracketed) and intron retention (arrows). qRT-PCR using primers that detect the presence of $5^{\prime}$ and $3^{\prime}$ splice junctions in Col7a1 further confirmed the RNA-seq data. Quantification of the qRT-PCR data is shown in F. Statistics uses 2-tailed Student's $t$ test, $n=3$.

Indeed, RNA-seq transcriptome analyses identify more target genes that are differentially expressed and show evidence of splicing defects in the FUS-R521C mice (Figure 9). Many are related to extracellular matrix organization, ion channels, and synaptic functions (Supplemental Table 1). The identification of these potential FUS-R521C target genes provides important insights into the dendritic and synaptic phenotypes in FUS-R521C mice and explain why BDNF can only partially restore the dendritic phenotype in neurons expressing FUS-R521C (Figure 8). The RNA-seq approach also reveals many target genes in the extracellular matrix assembly GO categories (GO:0005581, GO:0005201, GO:0005578, and GO:0031012) that have also been shown to be transcriptional targets of DNA damage response genes CSB and HDAC1 (43) and are frequently misregulated and misspliced in the motor neurons of SALS patients (54). Although these results are correlative, they raise the interesting possibility that the recruitment of FUS, HDAC1, and CSB may constitute a critical step in the repair of damaged DNA in FALS caused by FUS mutations and in SALS.

While our results highlight the inherent pathogenic properties of FUS-R521C mutation in DNA damage response/repair and RNA splicing, one potential caveat is that increased expression of WT FUS or mutant FUS-R521C proteins may interfere with physiological functions of endogenous FUS. For instance, expressing WT FUS in cultured cortical neurons causes dosedependent dendritic growth retardation and branching reduction (Figure 8, G-I, and Supplemental Figure 8C). Intriguingly, transgenic mice expressing WT FUS have been reported to show features of neurodegeneration in spinal motor neurons (55). In contrast, a similar transgenic approach to expressing WT FUS in rats results in no obvious neurological deficits, whereas transgenic rats expressing FUS-R521C do show neuropathological features similar to those of our FUS-R521C transgenic mice (56). The potential dose-dependent effects of WT FUS are reminiscent of the recent findings that WT FUS proteins are extremely likely to form protein-RNA complexes in hydrogel assays in a concentration-dependent fashion (57). Hence, it is conceivable that higher abundance of WT FUS proteins in neurons could potentially interfere with the physiological functions of endogenous FUS proteins. With the availability of the WT FUS transgenic mice (55), it will be interesting to determine whether excessive WT FUS may also interfere with RNA splicing in target genes that are similar or completely different from those in FUS-R521C transgenic mice.

One remarkable feature in the spinal cord of FUS-R521C transgenic mice is the upregulation of genes that are functionally related to immune response, complement activation, and chemotaxis (Figure 9B and Supplemental Table 1). These findings confirm the pronounced microgliosis in the FUS-R521 spinal cord (Supplemental Figure 3). Since neither WT FUS nor FUS-R521C proteins can be detected in the microglia (Supplemental Figure 2), these results support the idea that non-cell autonomous mechanisms involving activation of microglia may contribute to the neurodegeneration in $\operatorname{ALS}(58,59)$. Alternatively, the defects in DNA damage repair and RNA splicing caused by mutant FUS-R521C may occur in glia cells, which promote astroglial activation and/ or degeneration of oligodendroglia, further contributing to the degeneration of spinal motor neurons in FUS-R521C mice. Finally, our RNA-seq results in the spinal cord of FUS-R521C mutants show perturbations in the expression or splicing of several key components in the extracellular matrix, including members of the collagen and cadherin gene families, which regulate the specificity of axonal projection and target innervation $(45,46)$. Similar targets have also been identified in the fus morpholino-treated Xenopus morphants (60), suggesting that FUS-R521C phenotype may recapitulate certain transcriptional and RNA splicing defects in FUS loss-of-function mutants.

\section{Methods}

Mice. The FUS-R521C transgenic mice were generated using Syrian hamster prion promoter driving the expression of FLAG-tagged human FUS-R521C cDNA (gift from Stanley Prusiner, Institute of Neurodegenerative Disease, UCSF) (22). Two independent male founders (no. 3313 and no. 3303) were obtained from the pronuclear injections in B6SJL oocytes. The N1F1 progenies, generated by mating both founders with C57BL/ 6 female mice, showed comparable expression levels of FLAG-tagged FUS-R521C proteins in brain and spinal cord (Figure 2, A and B, $n=3$ ) and developed similar neurological abnormalities. Since the progenies from line no. 3313 had a higher propensity of developing disease and dying from their diseases, the N1F1 progenies from this line were used exclusively in this study. The mating scheme to generate N1F1 and to expand the N2F2 and N2F3 generations of the FUS-R521C transgenic mice is described in Supplemental Figure 2A. The transmission of the FUS-R521C transgene followed Mendelian inheritance for both N2F2 $(n=16)$ and N2F3 $(n=51)$. The disease onset and survival of the N2F2 and N2F3 FUS-R521C mice were nearly identical, supporting the successful propagation of the genotype and phenotype in these progenies (Supplemental Figure 1, C and D). The SOD $1^{\mathrm{G} 93 A}$ transgenic mice were obtained from the Jackson Laboratory. Animal husbandry followed guidelines established by the UCSF and San Francisco VA Medical Center IACUC and the NIH.

Histology, IHC, and in situ bybridization. Histopathology analyses were performed in mice that reached the disease end-stage at between 1 and 3 months of age. In each set of experiments, the nontransgenic littermates were used as control (referred to as control throughout the manuscript). Mice were euthanized and perfused with $4 \%$ paraformaldehyde (PFA). Brain and spinal cord tissues were dissected and post-fixed with $4 \%$ PFA overnight, followed by incubation with $30 \%$ sucrose solution and embedding in OCT. Immunostaining was performed in $40 \mu \mathrm{m}$ free-floating sections. FISH for Bdnf mRNA was performed in sections from control and FUS-R521C mice using cRNA probes that recognized either the coding sequence (CDS) or the $3^{\prime}$ UTR of the Bdnf gene (gifts from Baoji Xu, The Scripps Research Institute, Jupiter, Florida, USA) (30). 

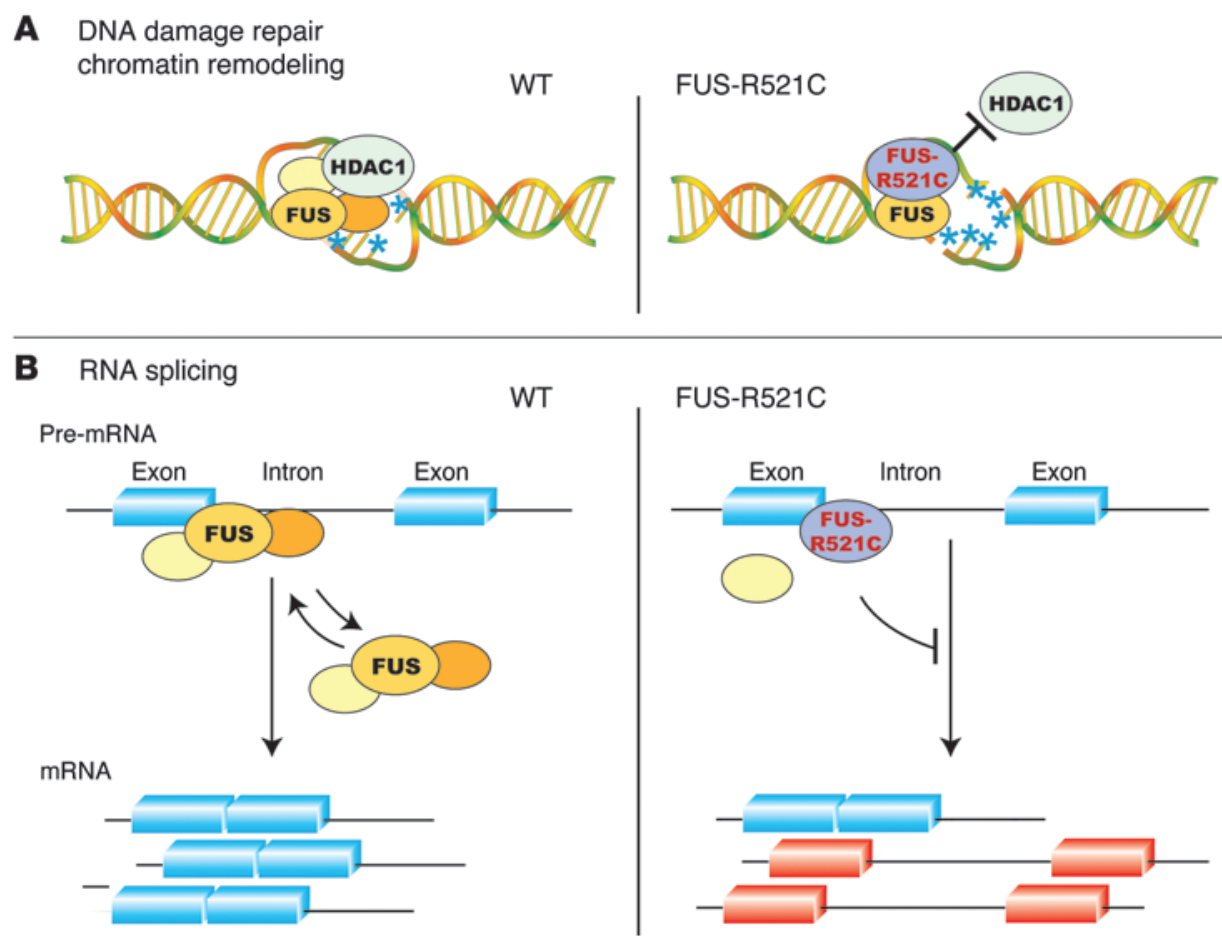

FUS-R521C

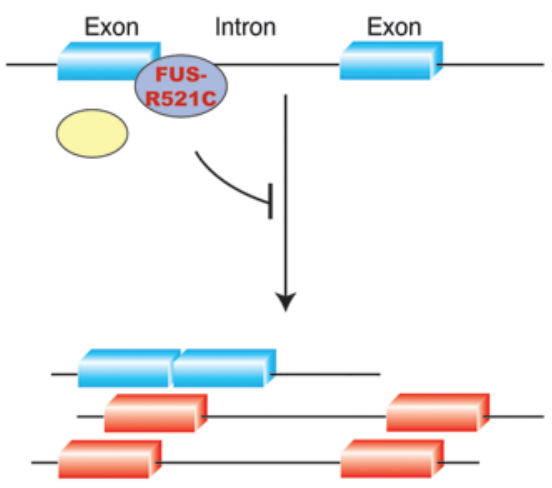

\section{Figure 10}

Proposed working models for the dominant inhibitory effects of FUS-R521C on DNA damage response/repair machinery and RNA splicing. (A) WT FUS is rapidly recruited to DNA damage foci caused by double-stranded breaks, where it interacts with chromatin remodeling factor HDAC1. Although FUS-R521C can still be recruited to DNA damage foci, it fails to interact with HDAC1. As a consequence, neurons in FUS-R521C transgenic mice show increased DNA damage (indicated by blue asterisks and the presence of double-stranded breaks). (B) Results from CLIP-RTPCR and protein-RNA interactions in EMSA assays show that both WT FUS and FUS-R521C can interact with selective oligoribonucleotides from Bdnf exon-intron boundaries. Whereas the equilibrium of WT FUS-RNA interactions appears to be more dynamic, FUS-R521C tends to form more stable protein-RNA complexes that are more difficult to dissociate.

Primary antibodies included cleaved caspase 3 (1:200, no. 9664; Cell Signaling), ChAT (1:300, AB144p; Millipore), Ctip2 (1:1,000, AB18465; Abcam), FLAG-M2 (1:1,000, F3165; Sigma-Aldrich), FUS (polyclonal rabbit antibody, 1:1,000, HPA008784; Sigma-Aldrich; and monoclonal antibody (4H11), 1:200, sc-47711; Santa Cruz Biotechnology Inc.), Olig2 (1:200, AB9610; Millipore), GFAP (1:5,000, Z0334; DAKO), H2AX (1:100, NB 100-79967; Novus Biologicals), $\gamma \mathrm{H} 2 \mathrm{AX}$ (1:200, 05-636; Millipore), HDAC1 (A-7) (1:200, SC-74563; Santa Cruz Biotechnology Inc.), Iba-1 (1:50,000,\#019-19741; Wako), MAP2 (1:500, AB11267; Abcam), and synaptophysin (1:1,000, \#5768; Sigma-Aldrich). Staining for ChAT required antigen retrieval treatment, which included incubating tissue sections in $10 \mathrm{mM}$ sodium citrate ( $\mathrm{pH} 6.0$ ) at $95^{\circ} \mathrm{C}$ for 15 to 20 minutes. The immunostaining was developed using either DAB technique or appropriate species-specific secondary antibodies conjugated with Alexa Fluor 488, Alex Fluor 568, or Alexa Fluor 636. Bright field and immunofluorescent images were captured using an Olympus BX53 microscope and a Leica LMS510 confocal microscope (510 Microimaging; Carl Zeiss), respectively. Quantification for SIPT per area was performed according to previously published protocol (61).

To determine the effects of FUS-R521C in the synthesis of BDNF, neurons were prepared from both control and FUS-R521C embryos and cultured for 14 days. The FISH images in cultured neurons were captured using a Zeiss LSM510 confocal microscope ( $\times 63$ oil objective, zoom $=1)$ with AIM version 4.2 software. Confocal images were imported to NIH ImageJ version $1.44 \mathrm{o}$ software, and the $B d n f$ mRNA signals were quanti- fied in the soma and dendrites for each neuron. The same images were imported to Neurolucida software for tracing along the dendrites. A virtual Zeiss $\times 63$ lens was added to the software and calibrated to the image scale bar. Each neuron tracing underwent Sholl analysis at 5 - $\mu \mathrm{m}$ radii from the cell body to determine the distribution of $B d n f$ mRNA throughout the dendrites. Quantifications of $B d n f$ mRNA signals were presented in graphs at matched radii using GraphPad Prism version 5.0c.

$E M$. Mice were perfused with 4\% PFA and tissue collected from the C1C3/L1-L3 spinal cord and sensorimotor cortex. Collected tissue was fixed overnight in 4\% PFA, sectioned using a Leica vibratome in cold PBS at 500 $\mu \mathrm{m}$ thick, and fixed in $0.0625 \%$ glutaraldehyde $/ 0.01 \% \mathrm{PFA} / 0.1 \mathrm{M}$ sodium cacodylate buffer ( $\mathrm{pH}$ 7.4). Following the fixation, the tissue was processed through $2 \%$ osmium tetroxide and $4 \%$ uranyl acetate, then dehydrated and embedded in Eponate 12 resin (Ted Pella Inc.). Ultra-thin sections were cut at $1-\mu \mathrm{m}$ thick, collected on copper grids, and imaged in a Phillips Tecnai10 transmission electron microscope using FEI software.

For spinal motor neurons, we surveyed 59 high-magnification fields from 27 neurons in 3 controls and 62 fields from 26 neurons in 3 FUS$\mathrm{R} 521 \mathrm{C}$ mice. These images gave rise to 76 terminals and 274 individual postsynaptic densities in controls and 67 terminals and 187 postsynaptic densities in FUS-R521C mice. For pyramidal neurons in layers 4 and 5 of the sensorimotor cortex, we surveyed 54 high-magnification fields from 3 controls and 58 fields from 3 FUS-R521C mice. These images gave rise to 47 terminals and 286 individual postsynaptic densities in controls and 57 terminals and 355 postsynaptic densities in FUS-R521C mice. 
Cortical neuron cultures. Primary cortical neurons were prepared from E17.5 WT CD-1 or FUS-R521C transgenic mice and cultured on polylysine-coated coverslips at a density of $5 \times 10^{5}$ cells/well. At DIV 7, neurons were transfected using Lipofectamine 2000 with plasmids that carry the cDNA construct for GFP, WT FUS, FUS-R521C, or FUS-P525L with Tandem tomato (tdTomato) cDNA. For in situ hybridization, neurons were fixed in $4 \%$ PFA at 7 days after transfection. For analyses of dendritic arborization, neurons were fixed with $4 \%$ PFA at 1,4 , or 7 days after transfection. Dendritic branches and length were quantified using Neurolucida Explorer version 4.71 .1 software at $5-\mu \mathrm{m}$ interval radii (MicroBrightField Inc.).

Golgi staining, neurolucida tracing, and quantification of dendrites and dendritic spines. The Golgi-Cox impregnation stains were performed using the FD Rapid GolgiStain Kit according to the manufacturer's instructions (MTR Scientific Inc.). Tracing of dendritic branching and total dendrite length was performed in Golgi-stained spinal motor neurons and pyramidal neurons from layers 4 to 5 of the sensorimotor cortex using the Olympus BX51 microscope, $\times 60$ oil objective, guided by Neurolucida software (version 8.26.1, MicroBrightField Inc.). Dendrites and dendritic spines were identified and underwent $3 \mathrm{D}$ reconstruction using Neurolucida to determine their density and position under microscope. This allowed the users a complete and impartial assessment of the dendritic spine density using Neurolucida Explorer. For spinal motor neurons, we analyzed the dendrites from 27 neurons from 3 age-matched controls and 26 neurons from 3 FUS-R521C mice. Identical numbers of neurons were analyzed for the dendrite phenotype in the pyramidal neurons in layers 4 and 5 of the sensorimotor cortex. Sholl analysis was performed on tracings in Neurolucida Explorer version 4.71.1 software at $5-\mu \mathrm{m}$ interval radii. Graphs representing mean \pm SEM and statistics were generated using GraphPad Prism version 5.0c. To characterize the spine phenotype in the pyramidal neurons in the sensorimotor cortex, we used Neurolucida to quantify the total numbers of spines and mature spines (mushroom-shaped morphology) starting at a distance greater than $30 \mu \mathrm{m}$ away from the soma along the apical dendrite and its secondary branches over a distance greater than $100 \mu \mathrm{m}$.

Western blots and immunoprecipitation. Total protein lysates were prepared by adding $1 \%$ NP-40 lysis buffer to brain and spinal cord tissues. For immunoprecipitation, equal amounts of proteins were precleared with protein $\mathrm{A} / \mathrm{G}$ agarose and incubated with primary antibody overnight. The protein $A / G$ agarose beads were added to protein antibody mixtures to perform pull-down. Western blots were carried out with different primary antibodies to detect FUS (1;1,000, HPA008784; Sigma-Aldrich), FLAG (1:1,000, F7425; Sigma-Aldrich), actin (1:5,000, CP01; Calbiochem), BDNF (1:1,000, SAB1402127; Sigma-Aldrich), c-Ret (1:1,000, sc-13104; Santa Cruz Biotechnology Inc.), phospho-c-Ret (p-Tyr1062, 1:1,000, sc-20252; Santa Cruz Biotechnology Inc.), phospho-TrkB (1:500, gift from Moses Chao, New York University, New York, New York, USA), or TrkB (1:500, \#4606; Cell Signaling).

qRT-PCR and CLIP-qRT-PCR. Total RNA prepared from brains and spinal cords of controls and FUS-R521C mice were used to detect the retention of intronic sequences in $B d n f$ mRNA using qRT-PCR, with the PCR primers designed to recognize the $5^{\prime}$ splice junctions on the noncoding exons of the $B d n f$ gene and exon-intron boundaries in the Col7a1 gene (Supplemental Table 2). One-step RT-PCR was performed with 100 ng RNAs, using the Brilliant II SYBR Green QRT-PCR Master Mix Kit (Agilent Technologies) according to the manufacturer's instructions.

CLIP-qRT-PCR was performed as described in the previously published protocols, with mild modifications (62). Briefly, $100 \mathrm{mg}$ brain tissue in PBS was fixed with $1 \%$ formaldehyde for 10 minutes. After 5 minutes quench with $0.25 \mathrm{M}$ glycine, samples were washed with ice-cold PBS 3 times, fol- lowed by treatment with RIPA buffer (50 mM Tris-Cl, pH 7.5, 1\% NP-40, $0.5 \%$ sodium deoxycholate, $0.05 \% \mathrm{SDS}, 1 \mathrm{mM}$ EDTA, $150 \mathrm{mM} \mathrm{NaCl}$, and protease inhibitors). After 3 rounds of 20-second sonication (Fisher Sonic Dismembrator, model 300; Fisher Scientific), cell debris was removed by centrifugation. Proteins $(1 \mathrm{mg})$ were precleared with protein agarose A/B and incubated with FUS primary antibody (Sigma-Aldrich) overnight. We chose this antibody because of it ability to recognize both WT FUS and mutant FUS-R521C proteins, thus providing a clear advantage to performing a side-by-side comparison of the binding of WT and mutant FUS proteins to endogenous $B d n f$ RNA. Antibodies were captured with agarose beads and then extensively washed with stringent washing buffer $(50 \mathrm{mM}$ Tris-Cl, $\mathrm{pH} 7.5,1 \% \mathrm{NP}-40,1 \%$ sodium deoxycholate, $0.1 \%$ sodium dodecyl sulfate, $1 \mathrm{mM}$ EDTA, $1 \mathrm{M} \mathrm{NaCl}, 1 \mathrm{M}$ urea, $0.2 \mathrm{mM}$ phenylmethylsulfonyl fluoride [PMSF]). The beads were resuspended in $100 \mathrm{ml}$ of $50 \mathrm{mM}$ Tris-Cl, $\mathrm{pH}$ 7.0, 5 mM EDTA, $10 \mathrm{mM}$ DTT, 1\% SDS. Cross-linking was reversed at $70^{\circ} \mathrm{C}$ for 45 minutes. RNA was extracted using Trizol, treated with DNase, and then subjected to qRT-PCR.

FPG assays. The FPG DNA damage assay was performed as previously described $(21,63)$. Briefly, the genomic DNA was isolated with the Qiagen DNeasy Blood \& Tissue Kit (no. 69504) with minor modifications, including all the buffers being purged with nitrogen for 10 minutes and supplemented with phenyl-tert-butyl nitrone (PBN); overnight digestion was carried out at $37^{\circ} \mathrm{C}$ to prevent in vitro oxidation during the DNA isolation process. Then $1.0 \mu \mathrm{g}$ of total DNA from each samples was incubated with formamidopyrimidine glycosylase (FPG; New English Biolabs), a bacterial $\mathrm{N}$-glycosylase and AP-lyase, to excise 8-oxoguanine and create DNA breaks. qPCR followed for examining the extent of DNA damage. Primers used in this study are listed in Supplemental Table 2.

EMSA. EMSA was performed according to a published protocol with some modifications (36). RNA oligos were end-labeled using adenosine $5^{\prime}$-triphosphate $\left(\left[\gamma^{-32} \mathrm{P}\right]\right)$ and polynucleotide kinase (M0201S; New England Biolabs) (Supplemental Table 2). The labeled oligos were purified using Microspin G-25 column (27-5325-01; GE Healthcare). The ${ }^{32}$ P-labeled RNAs were incubated with purified protein in $20 \mu$ l of RNA-binding buffer containing $50 \mathrm{mM} \mathrm{KCl}, 20 \mathrm{mM}$ Hepes, pH 7.5, 15\% glycerol, and $100 \mathrm{mg} / \mathrm{ml}$ tRNA for 30 minutes on ice. For competition experiments, nonradioactive probes were used at 10-, 25-, 100-, 200-, and 400-fold molar excess. Binding reactions were loaded onto $5 \%$ native polyacrylamide gels in $0.5 \times \mathrm{TBE}$ buffer (0.089 M Tris borate, $\mathrm{pH} 8,0.01 \mathrm{mM}$ EDTA) and run at 100 volts for 3 hours at $4^{\circ} \mathrm{C}$.

RNA-seq methods. Preparation of mRNA, double-stranded cDNA preparation, and ligation of barcode-containing adaptors were performed using the TruSeq SBS Kit V3 - HS according to the manufacturer's protocol (Illumina). Validation of DNA fragment size was performed using an Agilent Technologies 2100 Bioanalyzer, and DNA concentration was determined by quantitative PCR, following the manufacturer's protocol (Kapa Biosystems). The DNA library was sequenced on an Illumina GAIIX using a 100-nt paired-end read strategy following the manufacturer's protocol. The sequences were trimmed to $85 \mathrm{nt}$ and mapped to the mouse genome using CASAVA (Illumina) for assessment of sequence quality. The barcoded reads were separated, allowing for 2 mismatches in the barcode tag, and analyzed for their base composition. After preprocessing, the first 9 bases and the last 6 bases were removed from the beginning of all the reads. For de novo identification of junctions, the preprocessed reads were put into SpliceMap 3.3.6 and aligned to the mouse reference genome (44). Due to the size of the processed reads ( $85 \mathrm{nt}), 3$ mismatches were allowed in SpliceMap. For comparisons of the differences between control and FUS-R521C mice, 3 replicates were combined for each genotype.

Functional GO analyses. To characterize the extent of differentially expressed genes in RNA-seq, we used DESeq, an R package designed to analyze high-throughput sequencing assays for differential expression. Based 
on the DESeq analyses, the genes that show differential expression by RNAseq, were uploaded onto DAVID bioinformatics resources 6.7. The functional annotation chart and clustering analysis modules were employed to determine gene-term enrichment scores.

Unedited gels. See complete unedited gels in the supplemental material.

Statistics. Results are mean \pm SEM unless otherwise stated. The statistics of Sholl analyses in Figures 3 and 4 used 2-way repeated measures ANOVA and 2-tailed Student's $t$ test for dendritic length and number of branch points. Statistics used 2-tailed Student's $t$ test, except for the cumulative frequency histogram of synaptic density in Figure 4P, which used 2-tailed Mann-Whitney test. $P$ values of less than 0.05 were considered significant. Tests were performed with InStat of GraphPad Software.

Study approval. All animal procedures were conducted in strict compliance with the NIH Guide for the Care and Use of Laboratory Animals (8th edition. Revised 2011) as adopted by the NIH and were approved by UCSF and the San Francisco VA Medical Center.

\section{Acknowledgments}

We thank Moses Chao for the phospho-TrkB antibody; Baoji Xu for $B d n f$ in situ probes; Jimmy Perrott, Sol Katzman, and Nader
Pourmand at the UCSC Genome Technology Center for help with RNA-seq analyses; Sarah Bowman for the artwork in Figure 10; and members of the Huang lab for many helpful discussions. This work has been supported by grants from the NIH (OD010927 and OD011915 to E.J. Huang; NS078839 to L.H. Tsai; and HG005717 to K.F. Au), the Department of Veterans Affairs (BX001108 and BX001625 to E.J. Huang), and the Muscular Dystrophy Association (MDA217592 to E.J. Huang). H. Qiu is a recipient of the UCSF GEMS-CTSI Scholar Award, H. Lui is a recipient of the UCSF-Amgen Fellowship and the Irene and Eric Simon Brain Research Foundation Fellowship, and W.Y. Wang is supported by the Simons Foundation.

Received for publication August 20, 2013, and accepted in revised form November 27, 2013.

Address correspondence to: Eric J. Huang, Department of Pathology, University of California San Francisco, 505 Parnassus Avenue, San Francisco, California 94143, USA. Phone: 415.476.8525; Fax: 415.476.6206; E-mail: eric.huang2@ucsf.edu.
1. Kwiatkowski TJ Jr, et al. Mutations in the FUS/ TLS gene on chromosome 16 cause familial amyotrophic lateral sclerosis. Science. 2009;323(5918):1205-1208.

2. Vance C, et al. Mutations in FUS, an RNA processing protein, cause familial amyotrophic lateral sclerosis type 6. Science. 2009;323(5918):1208-1211.

3. Mackenzie IR, et al. Distinct pathological subtypes of FTLD-FUS. Acta Neuropathol. 2011;121(2):207-218.

4. Huang EJ, et al. Extensive FUS-immunoreactive pathology in juvenile amyotrophic lateral sclerosis with basophilic inclusions. Brain Pathol. 2010;20(6):1069-1076

5. Kuroda M, et al. Male sterility and enhanced radiation sensitivity in TLS $(-/-)$ mice. EMBO J. 2000;19(3):453-462.

6. Hicks GG, et al. Fus deficiency in mice results in defective B-lymphocyte development and activation, high levels of chromosomal instability and perinatal death. Nat Genet. 2000;24(2):175-179.

7. Wang WY, et al. Interaction of FUS and HDAC1 regulates DNA damage response and repair in neurons. Nat Neurosci. 2013;16(10):1383-1391.

8. Dobbin MM, et al. SIRT1 collaborates with ATM and HDAC1 to maintain genomic stability in neurons. Nat Neurosci. 2013;16(8):1008-1015.

9. Munoz MJ, et al. DNA damage regulates alternative splicing through inhibition of RNA polymerase II elongation. Cell. 2009;137(4):708-720.

10. Cleaver JE, Lam ET, Revet I. Disorders of nucleotide excision repair: the genetic and molecular basis of heterogeneity. Nat Rev Genet. 2009;10(11):756-768.

11. Kornblihtt AR, de la Mata M, Fededa JP, Munoz MJ, Nogues G. Multiple links between transcription and splicing. Rna. 2004;10(10):1489-1498.

12. Moore MJ, Proudfoot NJ. Pre-mRNA processing reaches back to transcription and ahead to translation. Cell. 2009;136(4):688-700.

13. Reed R, Hurt E. A conserved mRNA export machinery coupled to pre-mRNA splicing. Cell. 2002;108(4):523-531.

14. Li Q, Lee JA, Black DL. Neuronal regulation of alternative pre-mRNA splicing. Nat Rev Neurosci. 2007;8(11):819-831.

15. Martin KC, Ephrussi A. mRNA localization: gene expression in the spatial dimension. Cell. 2009;136(4):719-730

16. Cooper TA, Wan L, Dreyfuss G. RNA and disease. Cell. 2009;136(4):777-793.

17. Wahl MC, Will CL, Luhrmann R. The spliceosome: design principles of a dynamic RNP machine. Cell.
2009;136(4):701-718

18. Hoell JI, et al. RNA targets of wild-type and mutant FET family proteins. Nat Struct Mol Biol. 2011;18(12):1428-1431.

19. Rogelj B, et al. Widespread binding of FUS along nascent RNA regulates alternative splicing in the brain. Sci Rep. 2012;2:603.

20. Graff J, et al. An epigenetic blockade of cognitive functions in the neurodegenerating brain. Nature. 2012;483(7388):222-226.

21 . Lu T, et al. Gene regulation and DNA damage in the ageing human brain. Nature. 2004; 429(6994):883-891.

22. Westaway D, et al. Degeneration of skeletal muscle, peripheral nerves, and the central nervous system in transgenic mice overexpressing wild-type prion proteins. Cell. 1994;76(1):117-129.

23. Sun $Z$, et al. Molecular determinants and genetic modifiers of aggregation and toxicity for the ALS disease protein FUS/TLS. PLoS Biol. 2011;9(4):e1000614.

24. Fujii R, et al. The RNA binding protein TLS is translocated to dendritic spines by mGluR5 activation and regulates spine morphology. Curr Biol. 2005;15(6):587-593.

25. Fan F, et al. ATF3 induction following DNA damage is regulated by distinct signaling pathways and over-expression of ATF3 protein suppresses cells growth. Oncogene. 2002;21(49):7488-7496.

26. Reyes NA, Fisher JK, Austgen K, VandenBerg S, Huang EJ, Oakes SA. Blocking the mitochondrial apoptotic pathway preserves motor neuron viability and function in a mouse model of amyotrophic lateral sclerosis. J Clin Invest. 2010;120(10):3673-3679.

27. Gurney ME, et al. Motor neuron degeneration in mice that express a human $\mathrm{Cu}, \mathrm{Zn}$ superoxide dismutase mutation. Science. 1994;264(5166):1772-1775.

28. Betley JN, et al. Stringent specificity in the construction of a GABAergic presynaptic inhibitory circuit. Cell. 2009;139(1):161-174.

29. Loerch PM, et al. Evolution of the aging brain transcriptome and synaptic regulation. PLoS One. 2008;3(10):e3329.

30. An JJ, et al. Distinct role of long $3^{\prime}$ UTR BDNF mRNA in spine morphology and synaptic plasticity in hippocampal neurons. Cell. 2008;134(1):175-187.

31. Rauskolb S, et al. Global deprivation of brainderived neurotrophic factor in the CNS reveals an area-specific requirement for dendritic growth. J Neurosci. 2010;30(5):1739-1749.

32. Gorski JA, Zeiler SR, Tamowski S, Jones KR.
Brain-derived neurotrophic factor is required for the maintenance of cortical dendrites. J Neurosci. 2003;23(17):6856-6865.

33. Liu QR, Lu L, Zhu XG, Gong JP, Shaham Y, Uhl GR. Rodent BDNF genes, novel promoters, novel splice variants, and regulation by cocaine. Brain Res. 2006;1067(1):1-12.

34. Pruunsild P, Kazantseva A, Aid T, Palm K, Timmusk T. Dissecting the human BDNF locus: bidirectional transcription, complex splicing, and multiple promoters. Genomics. 2007;90(3):397-406.

35. Kanai Y, Dohmae N, Hirokawa N. Kinesin transports RNA: isolation and characterization of an RNA-transporting granule. Neuron. 2004;43(4):513-525.

36. Lerga A, et al. Identification of an RNA binding specificity for the potential splicing factor TLS. $J$ Biol Chem. 2001;276(9):6807-6816.

37. Matsumoto T, et al. Biosynthesis and processing of endogenous BDNF: CNS neurons store and secrete BDNF, not pro-BDNF. Nat Neurosci. 2008;11(2):131-133.

38. Iwakura Y, Nawa H, Sora I, Chao MV. Dopamine D1 receptor-induced signaling through TrkB receptors in striatal neurons. J Biol Chem. 2008;283(23):15799-15806.

39. Mitchell JC, et al. Overexpression of human wildtype FUS causes progressive motor neuron degeneration in an age- dose-dependent fashion. Acta Neuropathol. 2013;125(2):273-288.

40. Anders S, Huber W. Differential expression analysis for sequence count data. Genome Biol. 2010;11(10):R106.

41. Dasen JS, Tice BC, Brenner-Morton S, Jessell TM. A Hox regulatory network establishes motor neuron pool identity and target-muscle connectivity. Cell. 2005;123(3):477-491.

42. Huang da W, Sherman BT, Lempicki RA. Systematic and integrative analysis of large gene lists using DAVID bioinformatics resources. Nat Protoc. 2009;4(1):44-57.

43. Newman JC, Bailey AD, Weiner AM. Cockayne syndrome group $B$ protein (CSB) plays a general role in chromatin maintenance and remodeling. Proc Natl Acad Sci U S A. 2006;103(25):9613-9618.

44. Au KF, Jiang H, Lin L, Xing Y, Wong WH. Detection of splice junctions from paired-end RNA-seq data by SpliceMap. Nucleic Acids Res. 2010;38(14):4570-4578.

45. Robles E, Baier H. Assembly of synaptic laminae by axon guidance molecules. Curr Opin Neurobiol. 2012;22(5):799-804. 
46. Sanes JR, Yamagata M. Many paths to synaptic specificity. Annu Rev Cell Dev Biol. 2009;25:161-195.

47. Xiao T, Baier H. Lamina-specific axonal projections in the zebrafish tectum require the type IV collagen Dragnet. Nat Neurosci. 2007;10(12):1529-1537.

48. Xiao T, Staub W, Robles E, Gosse NJ, Cole GJ, Baier H. Assembly of lamina-specific neuronal connections by slit bound to type IV collagen. Cell. 2011;146(1):164-176

49. Couthouis J, et al. A yeast functional screen predicts new candidate ALS disease genes. Proc Natl Acad Sci U S A. 2011;108(52):20881-20890.

50. Ishigakiß S, et al. Position-dependent FUS-RNA interactions regulate alternative splicing events and transcriptions. Sci Rep. 2012;2:529.

51. Liao GY, et al. Dendritically targeted Bdnf mRNA is essential for energy balance and response to leptin. Nat Med. 2012;18(4):564-571.

52. Kim D, et al. Deregulation of HDAC 1 by p $25 / \mathrm{Cdk} 5$ in neurotoxicity. Neuron. 2008;60(5):803-817.
53. Jackson SP, Bartek J. The DNA-damage response in human biology and disease. Nature. 2009; 461(7267):1071-1078.

54. Rabin SJ, et al. Sporadic ALS has compartmentspecific aberrant exon splicing and altered cellmatrix adhesion biology. Hum Mol Genet. 2010; 19(2):313-328.

55. Mitchell JC, et al. Overexpression of human wildtype FUS causes progressive motor neuron degeneration in an age- and dose-dependent fashion. Acta Neuropathol. 2013;125(2):273-288.

56. Huang C, et al. FUS transgenic rats develop the phenotypes of amyotrophic lateral sclerosis and frontotemporal lobar degeneration. PLoS Genet. 2011;7(3):e1002011.

57. Kato M, et al. Cell-free formation of RNA granules: low complexity sequence domains form dynamic fibers within hydrogels. Cell. 2012;149(4):753-767.

58. Boillee S, Vande Velde C, Cleveland DW. ALS: a disease of motor neurons and their nonneuronal neighbors. Neuron. 2006;52(1):39-59.

59 . Boillee $S$, et al. Onset and progression in inherited ALS determined by motor neurons and microglia. Science. 2006;312(5778):1389-1392.

60. Dichmann DS, Harland RM. fus/TLS orchestrates splicing of developmental regulators during gastrulation. Genes Dev. 2012;26(12):1351-1363.

61. Chin J, Palop JJ, Yu GQ, Kojima N, Masliah E, Mucke L. Fyn kinase modulates synaptotoxicity, but not aberrant sprouting, in human amyloid precursor protein transgenic mice. J Neurosci. 2004;24(19):4692-4697.

62. Niranjanakumari S, Lasda E, Brazas R, GarciaBlanco MA. Reversible cross-linking combined with immunoprecipitation to study RNA-protein interactions in vivo. Methods. 2002;26(2):182-190.

63. O'Callaghan N, Baack N, Sharif R, Fenech M. A qPCR-based assay to quantify oxidized guanine and other FPG-sensitive base lesions within telomeric DNA. Biotechniques. 2011;51(6):403-411. 